J. DIFFERENTIAL GEOMETRY

82 (2009) 317-361

\title{
CONSTRUCTIONS OF SMALL SYMPLECTIC 4-MANIFOLDS USING LUTTINGER SURGERY
}

\author{
Scott BaldRidge \& Paul Kirk
}

\begin{abstract}
Luttinger surgery is used to produce minimal symplectic 4manifolds with small Euler characteristics. We construct a minimal symplectic 4-manifold which is homeomorphic but not diffeomorphic to $\mathbb{C P}^{2} \# 3 \overline{\mathbb{C P}}^{2}$, and which contains a genus two symplectic surface with trivial normal bundle and simply-connected complement. We also construct a minimal symplectic 4-manifold which is homeomorphic but not diffeomorphic to $3 \mathbb{C P}^{2} \# 5 \overline{\mathbb{C P}}^{2}$, and which contains two disjoint essential Lagrangian tori such that the complement of the union of the tori is simply-connected.

These examples are used to construct minimal symplectic manifolds with Euler characteristic 6 and fundamental group $\mathbb{Z}, \mathbb{Z}^{3}$, or $\mathbb{Z} / p \oplus \mathbb{Z} / q \oplus \mathbb{Z} / r$ for integers $p, q, r$. Given a group $G$ presented with $g$ generators and $r$ relations, a symplectic 4-manifold with fundamental group $G$ and Euler characteristic $10+6(g+r)$ is constructed.
\end{abstract}

\section{Introduction}

In this article we construct a number of small (as measured by the Euler characteristic $e$ ) simply connected and non-simply connected smooth 4-manifolds which admit symplectic structures. Specifically, we construct examples of:

- A minimal symplectic manifold $X$ homeomorphic but not diffeomorphic to $\mathbb{C P}^{2} \# 3 \overline{\mathbb{C P}}^{2}$ containing symplectic genus 2 surface with simply connected complement and trivial normal bundle, and a disjoint nullhomologous Lagrangian torus (Theorem 13).

- A minimal symplectic manifold $B$ homeomorphic but not diffeomorphic to $3 \mathbb{C P}^{2} \# 5 \overline{\mathbb{C P}}^{2}$ containing a disjoint pair of symplectic tori with simply connected complement and trivial normal bundle (Theorem 18). This provides a smaller substitute for the elliptic

The first author gratefully acknowledges support from the NSF grant DMS0507857 and NSF Career Grant DMS-0748636. The second author gratefully acknowledges support from the NSF grant DMS-0604310.

Received 03/01/2007. 
surface $E(1)$ in many 4-dimensional constructions.

- A minimal symplectic manifold $X_{1}$ with fundamental group $\mathbb{Z}$, Euler characteristic $e\left(X_{1}\right)=6$, signature $\sigma\left(X_{1}\right)=-2$ containing a symplectic torus $T$ with trivial normal bundle such that the inclusion $X_{1}-T \subset X_{1}$ induces an isomorphism on fundamental groups and so that the inclusion $T \subset X_{1}$ kills one generator of $\pi_{1}(T)$ (Theorem 22). This also provides a smaller substitute for $E(1)$ when only one generator is to be killed.

Variations on these constructions quickly provide many more examples of small simply connected minimal symplectic manifolds, including manifolds homeomorphic but not diffeomorphic to $\mathbb{C P}^{2} \# 5 \overline{\mathbb{C P}}^{2}$, $\mathbb{C P}^{2} \# 7 \overline{\mathbb{C P}}^{2}, 3 \mathbb{C P}^{2} \# 7 \overline{\mathbb{C P}}^{2}, 3 \mathbb{C P}^{2} \# 9 \overline{\mathbb{C P}}^{2}$, and $5 \mathbb{C P}^{2} \# 9 \overline{\mathbb{C P}}^{2}$. Constructions of small manifolds can also be found in $[\mathbf{2}, \mathbf{3}, \mathbf{6}, \mathbf{1 9}, \mathbf{2 7}, \mathbf{2 9}, \mathbf{3 2}$, $33,34]$.

The manifolds $X, B, X_{1}$ form building blocks which we use to prove a number of results, including the following.

- There exists an infinite family of pairwise non-diffeomorphic smooth simply connected manifolds each homeomorphic to $\mathbb{C P}^{2} \#$ $3 \overline{\mathbb{C P}}^{2}$.

- If a group $G$ has a presentation with $g$ generators and $r$ relations, then there exists a symplectic 4-manifold $M$ with fundamental group $G, e(M)=10+6(g+r)$ and $\sigma(M)=-2(g+r+1)$ (Theorem 24).

- For any pair of non-negative integers $m, n$ there exists a minimal symplectic manifold which is homeomorphic but not diffeomorphic to $(1+2 m+2 n) \mathbb{C P}^{2} \#(3+6 m+4 n) \overline{\mathbb{C P}}^{2}$ (Corollary 19).

- For any integers $p, q, r$, there exists a symplectic manifold $X_{p, q, r}$ with fundamental group $\mathbb{Z} / p \oplus \mathbb{Z} / q \oplus \mathbb{Z} / r$ with $e=6$ and $\sigma=-2$ (Corollary 30).

- If an abelian group $G$ is generated by $n$ elements with $n$ even, then there exists a symplectic 4-manifold with fundamental group $G$, $e=\frac{1}{2} n^{2}+\frac{19}{2} n+36$ and $\sigma=-\frac{5}{2} n-8$. (Theorem 31).

- For any non-negative integer $n$, there exists a symplectic 4-manifold with fundamental group free of rank $n, e=10$ and $\sigma=-2$. (Theorem 25).

- For any symplectic manifold $M$ containing a symplectic surface $H$ of genus 1 or 2 with trivial normal bundle so that the homomorphism $\pi_{1}(H) \rightarrow \pi_{1}(M)$ induced by inclusion is trivial, there exists infinitely many smooth manifolds $M_{n}$ with $\pi_{1}\left(M_{n}\right)=\pi_{1}(M)$, $e\left(M_{n}\right)=e(M)+2+4 \operatorname{genus}(H), \sigma\left(M_{n}\right)=\sigma(M)-2$, and the Seiberg-Witten invariants of $M_{n}$ are different from those of $M_{m}$ if $n \neq m$ (Corollary 21). 
We refer the reader to the body of the article for more precise statements of these theorems and further results. One particular feature of our constructions is that they contain nullhomologous Lagrangian tori for which the method of [10] allow us to produce families of infinitely many non-diffeomorphic but homeomorphic manifolds.

Our main tools are Luttinger and torus surgery $[\mathbf{2 1}, \mathbf{1}, \mathbf{1 3}]$, Gompf's symplectic sum construction [15], and, most importantly, the SeifertVan Kampen theorem, which we use to prove our central result, Theorem 11. This is then combined with Freedman's theorem [14] and fundamental results from Seiberg-Witten theory $[\mathbf{3 6}, \mathbf{3 7}, \mathbf{3 9}, \mathbf{2 3}]$ in the applications.

A problem which motivates our investigations concerns uniqueness of the diffeomorphism type of a symplectic manifold which has the smallest Euler characteristic among symplectic manifolds with a fixed fundamental group. For example, for the trivial group, the "symplectic Poincaré conjecture" (cf. [5]) asks whether a symplectic manifold homeomorphic to $\mathbb{C P}^{2}$ is diffeomorphic to $\mathbb{C P}^{2}$. Many constructions on 4 -manifolds are simpler to carry out when the Euler characteristic is large, and this has motivated the problem of finding interesting (e.g. exotic) simply connected or non-simply connected 4-manifolds with small Euler characteristic. As one works with smaller manifolds, it becomes difficult to alter the smooth structure without changing the fundamental group or destroying the existence of a symplectic structure.

Another question which motivates these results concerns, for a given group $G$, the gap between the smallest possible Euler characteristic of smooth 4-manifolds with fundamental group $G$, and the smallest possible Euler characteristic of symplectic 4-manifolds with fundamental group $G$, or the smallest possible Euler characteristic of complex surfaces with fundamental group $G$. For example, one can construct a smooth 4-manifold with fundamental group the finite cyclic group $\mathbb{Z} / n$ and $e=2$; this is the smallest possible. Corollary 30 establishes the existence of a symplectic 4-manifold with fundamental group $\mathbb{Z} / n$ and $e=6$, this is smallest currently known although it is possible that a smaller one exists. The smallest known complex surface with finite cyclic fundamental group has $e=10$.

The paper is organized as follows. In Section 2 we describe Luttinger surgery and calculate the fundamental group of the complement of some tori in the 4-torus. In Section 3 we construct the three main building blocks needed for all subsequent constructions. In Section 4 we prove our main result, Theorem 11, which computes the fundamental group (and all meridians and Lagrangian push offs) of the complement of six Lagrangian tori and a symplectic genus two surface in a certain symplectic manifold $Z$ satisfying $e(Z)=6, \sigma(Z)=-2$ and $H_{1}(Z)=\mathbb{Z}^{6}$. With this result in place we construct the simply connected examples 
described above and in Section 5 we construct the non-simply connected examples.

To the extent that the methods of the present article focus on quite involved calculations of fundamental groups, we take great care with our use of the Seifert-Van Kampen theorem, choice of representative loops, and choices of base points. Some of the fundamental group assertions we prove are perhaps not surprising. However, the introduction of unwanted conjugation at any stage can easily lead to a loss of control over fundamental groups, in particular leading to plausible but unverifiable calculations. Given the usefulness of our theorems and that such methods are not so common in 4-dimensional topology, we feel the care we take is justified.

Acknowledgments. The authors thank A. Akhmedov, R. Fintushel, C. Judge, C. Livingston, and J. Yazinsky for helpful discussions, and thank the referees for their careful reading and helpful suggestions.

\section{The fundamental group of the complement of some tori in the 4-torus}

2.1. Luttinger surgery. Given any Lagrangian torus $T$ in a symplectic 4-manifold $M$, the Darboux-Weinstein theorem [22] implies that there is a parameterization of a tubular neighborhood of $T, T^{2} \times D^{2} \cong$ $n b d(T) \subset M$, such that the image of $T^{2} \times\{d\}$ is Lagrangian for all $d \in D^{2}$. Choosing any point $d \neq 0$ in $D^{2}$ determines a push off $F_{d}: T \rightarrow T^{2} \times\{d\} \subset M-T$ called the Lagrangian push off or Lagrangian framing. Given any embedded curve $\gamma \subset T$, its image $F_{d}(\gamma)$ is called the Lagrangian push off of $\gamma$. The smooth isotopy class of the Lagrangian push off $F_{d}: T \rightarrow M-T$ depends only on the symplectic structure in a neighborhood of $T$. As is common we will abuse terminology slightly and call the isotopy class of $F_{d}(\gamma)$ for any $d \neq 0$ the Lagrangian push off of $\gamma$. Any curve isotopic to $\{t\} \times \partial D^{2} \subset \partial(n b d(T))$ will be called a meridian of $T$ and denoted by $\mu$.

Fix $d \in \partial D$. If $x, y$ are loops in $T$ generating $H_{1}(T)$, let $m=F_{d}(x)$ and $\ell=F_{d}(y)$. Then the triple $\mu, m, \ell$ generate $H_{1}(\partial(n b d(T)))$. Since the 3 -torus has abelian fundamental group we may choose a base point $t$ on $\partial(n b d(T))$ and unambiguously refer to $\mu, m, \ell \in \pi_{1}(\partial(n b d(T)), t)$.

The push offs and meridians are used to specify coordinates for a $p / q$ torus surgery on $T$ along $\gamma$. This is the process of removing a tubular neighborhood of $T$ in $M$ and re-gluing it so that the embedded curve representing $\mu^{p} F_{d}(\gamma)^{q}$ bounds a disk. The diffeomorphism type of the resulting manifold depends only on the isotopy class of the identification $T^{2} \times D^{2} \rightarrow n b d(T)$, and not on the particular point $d$ or the specific choice of $\mu$. Its fundamental group is isomorphic to

$$
\pi_{1}(M-T) / N\left(\mu^{p} F_{d}(\gamma)^{q}\right)
$$


where $N\left(\mu^{p} F_{d}(\gamma)^{q}\right)$ denotes the normal subgroup generated by $\mu^{p} F_{d}(\gamma)^{q}$.

When the base point of $M$ is chosen off the boundary of the tubular neighborhood of $T$, the based loops $\mu$ and $\gamma$ are to be joined to the base point by the same path in $M-T$. Then Equation (1) holds with respect to this choice of basing.

Note that if one fixes generating curves $x, y$ on $T$, then the embedded curve $\gamma$ can be expressed in $\pi_{1}(T)$ in the form $\gamma=x^{a} y^{b}$ for some relatively prime pair of integers $a, b$. In that case the fundamental group of the manifold obtained by $p / q$ torus surgery on $T$ along $\gamma$ is

$$
\pi_{1}(M-T) / N\left(\mu^{p} m^{a q} \ell^{b q}\right)
$$

where, as above, $m=F_{d}(x)$ and $\ell=F_{d}(y)$.

The special case of $p=1, q=k$ is called $1 / k$ Luttinger surgery on $T$ along the embedded curve $\gamma \subset T$. This yields a symplectic manifold $([\mathbf{2 1}, \mathbf{1}])$. The symplectic form is unchanged away from a neighborhood of $T$. The fundamental group of the manifold obtained by $1 / k$ Luttinger surgery on $T$ along an embedded curve $\gamma$ is isomorphic to

$$
\pi_{1}(M-T) / N\left(\mu F_{d}(\gamma)^{k}\right)
$$

where $N\left(\mu F_{d}(\gamma)^{k}\right)$ denotes the normal subgroup generated by $\mu F_{d}(\gamma)^{k}$.

It is sometimes convenient to adopt the language of 3 -dimensional topology and call the process of gluing $T \times D^{2}$ to $M-n b d(T)$ a $1 / k$ Luttinger filling, or, more generally, a $p / q$ torus filling.

When $p \neq \pm 1$ there is no reason why the symplectic form should extend over the neighborhood of $T$, and typically the smooth manifold obtained by $p / q$ surgery admits no symplectic structure when $p \neq \pm 1$.

2.2. The complement of two Lagrangian tori in the product of two punctured tori. Let $\hat{H}$ and $\hat{K}$ denote a pair of 2-tori, endowed with the standard symplectic form. Removing an open disk from $\hat{H}$ and $\hat{K}$ yields punctured tori $H=\hat{H}-D$ and $K=\hat{K}-D$. View $H \times K$ as a codimension 0 symplectic submanifold of $T^{4}=\hat{H} \times \hat{K}$ with its standard product symplectic form. The product $H \times K$ should be considered as the complement of a tubular neighborhood of the (singular) union of two symplectic tori $\left(\hat{H} \times\left\{u_{K}\right\}\right) \cup\left(\left\{u_{H}\right\} \times \hat{K}\right) \subset \hat{H} \times \hat{K}$ (where $u_{H}$ and $u_{K}$ denote the centers of the disks removed.)

Choose a pair of curves $x, y$ representing a standard generating set for $\pi_{1}(H)$ and a pair of curves $a, b$ representing a standard generating set for $\pi_{1}(K)$. Let $X, Y$ be parallel push offs of $x$ and $y$ in $H$ and let $A_{1}, A_{2}$ be parallel push offs of $a$ in $K$, as illustrated in the following figure. Let $h$ be the intersection point of $x$ and $y$ and let $k$ be the intersection point of $a$ and $b$. Give $H \times K$ the base point $(h, k)$.

We define two disjoint tori $T_{1}, T_{2}$ in $H \times K$ as follows.

$$
T_{1}=X \times A_{1} \text { and } T_{2}=Y \times A_{2} .
$$



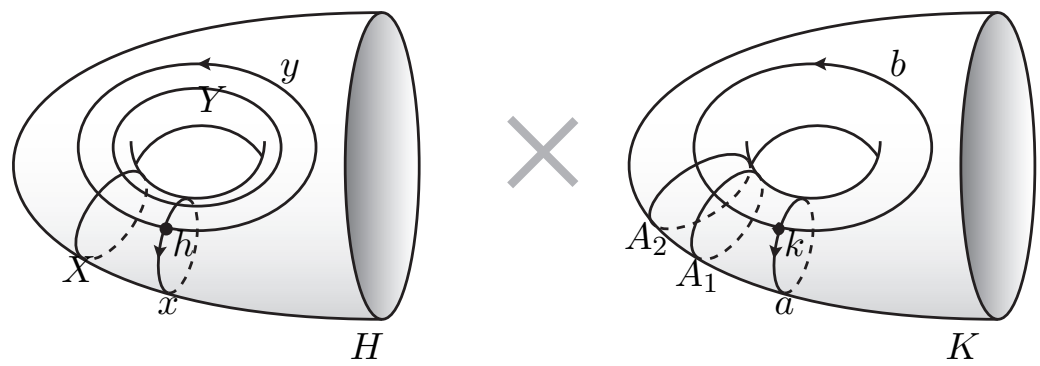

Figure 1. The surface $H \times K$.

Then the tori $T_{1}$ and $T_{2}$ are Lagrangian and the surfaces $H \times\{p\}$ and $\{q\} \times K$ are symplectic for any $p \in K$ and $q \in H$.

Let $A_{i}^{\prime}$ denote a push off of $A_{i}$ into $K-\left(A_{1} \cup A_{2}\right), i=1,2$. Then the parallel tori $T_{1}^{\prime}=X \times A_{1}^{\prime}$ and $T_{2}^{\prime}=Y \times A_{2}^{\prime}$ are Lagrangian, and so the Lagrangian push off of a curve on $T_{i}$ is its image in $H \times K-\left(T_{1} \cup T_{2}\right)$ using this push off $T_{i} \rightarrow T_{i}^{\prime}$. Sometimes it is preferable to use the push offs using the parallel tori $X^{\prime} \times A_{1}$ or $Y^{\prime} \times A_{2}$ where $X^{\prime}$ and $Y^{\prime}$ are parallel copies in $H$ of $X$ and $Y$. As we explained above, the manifolds resulting from torus and Luttinger surgery are well defined up to diffeomorphism.

The boundary of the tubular neighborhood of $T_{i}$ is a 3-torus. Therefore $H_{1}\left(\partial\left(n b d\left(T_{i}\right)\right)\right)=\mathbb{Z}^{3}$, with generating set $\left\{\mu_{i}, m_{i}, \ell_{i}\right\}$, where $\mu_{i}$ is the meridian and $m_{i}$ and $\ell_{i}$ the Lagrangian push offs of two generators of $H_{1}\left(T_{i}\right)$.

We specify notation for certain explicit loops in $H \times K$ based at $(h, k)$.

1) The loop $x \times\{k\}: I \rightarrow H \times\{k\}$ based at $(h, k)$ will be denoted simply by $x$. This loop misses $T_{1} \cup T_{2}$.

2) The loop $y \times\{k\}: I \rightarrow H \times\{k\}$ based at $(h, k)$ will be denoted by $y$. This loop misses $T_{1} \cup T_{2}$.

3) The loop $\{h\} \times a: I \rightarrow\{h\} \times K$ based at $(h, k)$ will be denoted by $a$. This loop misses $T_{1} \cup T_{2}$.

4) The loop $\{h\} \times b: I \rightarrow\{h\} \times K$ based at $(h, k)$ will be denoted by $b$. This loop misses $T_{1} \cup T_{2}$.

In $[\mathbf{6}$, Section 2] we proved the following theorem.

Theorem 1. There exist paths in $H \times K-\left(T_{1} \cup T_{2}\right)$ from the base point $(h, k)$ to the boundary of the tubular neighborhoods $T_{1} \times \partial D^{2}$ and $T_{2} \times \partial D^{2}$ with the following property.

Denote by $\mu_{i}, m_{i}, \ell_{i}$ the loops in $H \times K-\left(T_{1} \cup T_{2}\right)$ based at $(h, k)$ obtained by following the chosen path to the boundary of the tubular neighborhood of $T_{i}$, then following (respectively) the meridian of $T_{i}$ and the two Lagrangian push offs of the generators on $T_{i}$, then returning to the base point along the chosen path. 
Then in $\pi_{1}\left(H \times K-\left(T_{1} \cup T_{2}\right),(h, k)\right)$ :

$$
\mu_{1}=\left[b^{-1}, y^{-1}\right], m_{1}=x, \ell_{1}=a,
$$

and

$$
\mu_{2}=\left[x^{-1}, b\right], m_{2}=y, \ell_{2}=b a b^{-1} .
$$

where $x, y, a, b$ are the loops described above.

Moreover, $\pi_{1}\left(H \times K-\left(T_{1} \cup T_{2}\right),(h, k)\right)$ is generated by $x, y, a, b$ and the relations

$$
[x, a]=1,[y, a]=1,\left[y, b a b^{-1}\right]=1
$$

as well as

$$
[[x, y], b]=1,[x,[a, b]]=1,[y,[a, b]]=1
$$

hold in $\pi_{1}\left(H \times K-\left(T_{1} \cup T_{2}\right),(h, k)\right)$.

q.e.d.

The two important things to note in this theorem are, first, the homotopy class of the loops $x, y, a$, and $b$ based at $(h, k)$ generate $\pi_{1}\left(H \times K-\left(T_{1} \cup T_{2}\right),(h, k)\right)$. Second, the explicit expressions for $\mu_{i}, m_{i}, \ell_{i}$ allows us to list relations that hold in the fundamental group of the manifold obtained from torus surgery on the $T_{i}$ in $H \times K$. For example, the relations

$$
\left[b^{-1}, y^{-1}\right] x^{2} a^{6}=1 \text { and }\left[x^{-1}, b\right] b a^{-1} b^{-1}=1
$$

hold in the fundamental group of the manifold obtained from $H \times K$ by performing $1 / 2$ surgery on $T_{1}$ along $m_{1} \ell_{1}^{3}$ and $-1 / 1$ surgery on $T_{2}$ along $\ell_{2}$.

We will also need the following result.

Lemma 2. Consider the manifold L obtained from $T^{4}=\hat{H} \times \hat{K}$ by performing Luttinger surgeries on $T_{1}$ along $m_{1}$ and $T_{2}$ along either $m_{2}$ or $\ell_{2}$. Then $L$ is aspherical.

Proof. Suppose $L$ is obtained from $T^{4}$ by performing $1 / k_{1}$ Luttinger surgery on $T_{1}$ along $m_{1}$ and $1 / k_{2}$ Luttinger surgery on $T_{2}$ along $\gamma$, where $\gamma=m_{2}$ or $\ell_{2}$.

In the case when $\gamma=m_{2}$, it is straightforward to see that $L$ is diffeomorphic to $U \times S^{1}$, where $U$ is the 3-manifold that fibers over $S^{1}$ with fiber $\hat{H}$ and monodromy $D_{Y}^{k_{2}} \circ D_{X}^{k_{1}}$, where $D_{X}$ and $D_{Y}$ are the positive Dehn twists along $X$ and $Y$ in $\hat{H}$. This is explained carefully in $[\mathbf{1}, \mathrm{pg}$. 189]. Thus the universal cover of $L$ is $\mathbb{R}^{4}$.

In the case when $\gamma=\ell_{2}$, it is not hard to show (see [4]) that $L$ is diffeomorphic to a non-trivial $S^{1}$ bundle over $U$, where $U$ is the 3 manifold that fibers over $S^{1}$ with fiber $\hat{H}$ and monodromy $D_{X}^{k_{1}}$, and the first Chern class of the bundle is $k_{2} \cdot P D_{U}([Y])$ where $P D_{U}$ denotes Poincaré duality in $U$. Thus again the universal cover of $L$ is $\mathbb{R}^{4}$.

In either case $L$ is aspherical.

q.e.d. 
By symmetry, Lemma 2 holds as well if both surgeries are performed along $\ell_{i}$.

\section{Three small building blocks}

3.1. Our first and simplest building block is the symplectic manifold $W_{1}=\left(T^{2} \times S^{2}\right) \# 4 \overline{\mathbb{C P}}^{2}$, containing a symplectic genus 2 surface $F_{1}$ with trivial normal bundle.

We construct the surface $F_{1}$ by starting with the union of two parallel copies $T^{2} \times\left\{p_{1}\right\}, T^{2} \times\left\{p_{2}\right\}$ of the torus factor and one copy of $\{q\} \times S^{2}$ in $T^{2} \times S^{2}$. Each of these three surfaces is an embedded symplectic submanifold, and $\{q\} \times S^{2}$ intersects each of the tori in one point. We symplectically resolve the two double points (cf. [15]), to obtain a symplectic genus 2 surface $F_{1}$ of square $(2[T]+[S])^{2}=4$ in $T^{4}$. Recall that, topologically, symplectically resolving corresponds to locally replacing a pair of transversely intersecting discs by an annulus.

Blowing up $T^{2} \times S^{2}$ four times at points which lie on $F_{1}$ and taking the proper transform yields the desired $F_{1} \subset W_{1}$. The surface $F_{1}$ has trivial normal bundle and $W_{1}$ contains an embedded -1 sphere intersecting $F_{1}$ transversally in exactly one point.

Let $\phi: F_{1} \rightarrow W_{1}-n b d\left(F_{1}\right)$ be a push off of $F_{1}$, and choose a base point $w \in \phi\left(F_{1}\right)$. Since $W_{1}$ contains a -1 sphere intersecting $F_{1}$ transversally, the meridian of $F_{1}$ is nullhomotopic in $W_{1}-n b d\left(F_{1}\right)$. Hence the inclusion $W_{1}-n b d\left(F_{1}\right) \rightarrow W_{1}$ induces an isomorphism on fundamental groups by transversality and the Seifert-Van Kampen theorem.

The two circle coordinates of $T^{2}$ define classes $s, t \in H_{1}\left(W_{1}\right)$. Given any base point in $W_{1}$, we may unambiguously write $\pi_{1}\left(W_{1}\right)=\mathbb{Z} s+\mathbb{Z} t$, since $\pi_{1}\left(W_{1}\right)$ is abelian.

One can choose four loops $s_{1}, t_{1}, s_{2}, t_{2}$ on $\phi\left(F_{1}\right)$ based at $w$ which generate $\pi_{1}\left(\phi\left(F_{1}\right), w\right)$ and so that $\left[s_{1}, t_{1}\right]\left[s_{2}, t_{2}\right]=1$ in $\pi_{1}\left(\phi\left(F_{1}\right), w\right)$ in such a way that the composite

$$
\pi_{1}\left(F_{1}\right) \stackrel{\phi_{*}}{\longrightarrow} \pi_{1}\left(W_{1}-n b d\left(F_{1}\right)\right) \cong \pi_{1}\left(W_{1}\right) \cong H_{1}\left(W_{1}\right)
$$

takes $s_{1}$ to $s, s_{2}$ to $s^{-1}, t_{1}$ to $t$, and $t_{2}$ to $t^{-1}$. Thus we adopt the notation:

1) The loop $s_{1}: I \rightarrow \phi\left(F_{1}\right) \subset \partial\left(W_{1}-n b d\left(F_{1}\right)\right) \subset W_{1}-n b d\left(F_{1}\right)$ based at $w$ is a representative loop for the based homotopy class $s \in \pi_{1}\left(W_{1}, w\right)$.

2) The loop $t_{1}: I \rightarrow \phi\left(F_{1}\right) \subset \partial\left(W_{1}-n b d\left(F_{1}\right)\right) \subset W_{1}-n b d\left(F_{1}\right)$ based at $w$ is a representative loop for the based homotopy class $t \in \pi_{1}\left(W_{1}, w\right)$.

Then the following proposition holds.

Proposition 3. The symplectic surface $F_{1}$ intersects an embedded sphere transversally in one point and the inclusion $W_{1}-n b d\left(F_{1}\right) \subset W_{1}$ 
induces an isomorphism on fundamental groups. The inclusion $\phi\left(F_{1}\right) \subset$ $W_{1}-n b d\left(F_{1}\right)$ induces a surjection on fundamental groups.

Moreover, the loops $s_{1}, t_{1}, s_{2}, t_{2}$ on $\phi\left(F_{1}\right)$ can be chosen so that $\pi_{1}\left(W_{1}-n b d\left(F_{1}\right), w\right)=\mathbb{Z} s \oplus \mathbb{Z} t$, where $s, t$ are just the loops $s_{1}, t_{1}$ viewed as loops in $W_{1}-F_{1}$, and so that the inclusion $\phi\left(F_{1}\right) \rightarrow W_{1}-n b d\left(F_{1}\right)$ induces the homomorphism $s_{1} \mapsto s, t_{1} \mapsto t, s_{2} \mapsto s^{-1}$, and $t_{2} \mapsto t^{-1}$. Every -1 sphere in $W_{1}$ intersects $F_{1}$.

Proof. The assertions about the fundamental group are explained above.

The four exceptional spheres all meet $F_{1}$ since the blowup was performed on $F_{1}$. Denote by $T, S, E_{1}, E_{2}, E_{3}$, and $E_{4}$ the five generators of $H_{2}\left(W_{1}\right)$, where $T=T^{2} \times\{p\}, S=\{q\} \times S$, and the $E_{i}$ are the exceptional classes. Thus $F_{1}=2 T+S-E_{1}-E_{2}-E_{3}-E_{4}$. The Hopf exact sequence $\pi_{2}\left(W_{1}\right) \rightarrow H_{2}\left(W_{1}\right) \rightarrow H_{2}\left(\pi_{1}\left(W_{1}\right)\right) \rightarrow 0$ shows that the spherical classes are spanned by $S, E_{1}, E_{2}, E_{3}, E_{4}$ (since $T$ maps to the generator of $\left.H_{2}\left(\pi_{1}\left(W_{1}\right)\right)\right)$. Consideration of the intersection form shows that a -1 sphere must have the form $a S \pm E_{i}$. Then $\left(a S \pm E_{1}\right) \cdot F_{1}=2 a \pm 1 \neq 0$. Thus every -1 sphere intersects $F_{1}$.

q.e.d.

Suppose that $P$ is any symplectic 4-manifold containing a symplectic surface $G$ of genus 2 with trivial normal bundle. Then the symplectic sum, $S$, of $W_{1}$ and $P$ along $F_{1}$ and $G$ (cf. [15]) is a symplectic manifold described topologically as the union of $W_{1}-n b d\left(F_{1}\right)$ and $P-n b d(G)$ along their boundary using a fiber preserving diffeomorphism $F_{1} \times S^{1} \rightarrow G \times S^{1}$ of the boundary of their tubular neighborhoods. The diffeomorphism type of the manifold $S$ may depend on the choice of such a diffeomorphism, which can be specified up to isotopy by choosing trivializations of the tubular neighborhoods of $F_{1}$ and $G$ and a diffeomorphism $\phi: F_{1} \rightarrow G$. One then glues $W_{1}-n b d\left(F_{1}\right)$ to $P-\operatorname{nbd}(G)$ using the gluing diffeomorphism

$$
\begin{gathered}
\tilde{\phi}: \partial\left(W_{1}-n b d\left(F_{1}\right)\right)=F_{1} \times S^{1} \cong \partial(P-n b d(G))=G \times S^{1}, \\
\tilde{\phi}(f, z)=(\phi(f), z) .
\end{gathered}
$$

Then

$$
S=W_{1}-n b d\left(F_{1}\right) \cup_{\tilde{\phi}} P-n b d(G) .
$$

The symplectic sum is defined more generally when $G$ and $F$ have normal bundles with opposite Euler class, i.e. if $[G]^{2}=-[F]^{2}$. For our purposes it will suffice to consider symplectic sums along square zero surfaces. Moreover, the framings we use will either be explicit, or unimportant to the fundamental group calculations.

Assume that the base point $p$ of $P$ lies on $G$, and that $\phi: F_{1} \rightarrow G$ is base point preserving, $\phi(w)=p$. Denote by $N$ the subgroup of $\pi_{1}(P, p)$ normally generated by $\phi\left(s_{1} s_{2}\right), \phi\left(t_{1} t_{2}\right)$, and $\phi\left(\left[s_{1}, t_{1}\right]\right)$. Then 
Proposition 3 and the Seifert-Van Kampen theorem imply that $\pi_{1}(S, w)$ is a quotient of $\pi_{1}(P, p) / N$.

More generally, one can replace $W_{1}$ and $F_{1}$ by any appropriate pair $W, F$. We state this formally:

Lemma 4. Suppose the 4-manifold $W$ contains a genus 2 surface $F$ with trivialized normal bundle, and the 4-manifold $P$ contains a genus 2 surface $G$ with trivialized normal bundle. Let $\phi: F \rightarrow G$ be a diffeomorphism, and let $\tilde{\phi}=\phi \times I d: F \times S^{1} \rightarrow G \times S^{1}$.

Suppose that

1) F meets a sphere in $W$ transversally in one point,

2) The inclusion $F \rightarrow W$ induces a surjection on fundamental groups. Let

$$
S=(W-n b d(F)) \cup_{\tilde{\phi}}(P-n b d(G)) .
$$

Then there is a surjection

$$
\pi_{1}(P) \rightarrow \pi_{1}(S)
$$

whose kernel contains $\phi(r)$, for every loop $r$ in $\operatorname{ker} \pi_{1}(F) \rightarrow \pi_{1}(W)$.

q.e.d.

In our applications of this lemma we will typically use it to show $S$ is simply connected, or use it when $P$ is simply connected. In either of these cases base point issues will not matter. Notice also that choice of trivializations of the normal bundle do not affect the conclusion.

We state a similar but easier fact whose proof can be safely left to the reader.

Lemma 5. Suppose the 4-manifold $W$ contains a genus 2 surface $F$ with trivialized normal bundle, with $W-F$ simply connected. Let $P$ be a 4-manifold containing a genus 2 surface $G$ with trivialized normal bundle. Let $\phi: F \rightarrow G$ be a diffeomorphism, and let $\tilde{\phi}=\phi \times I d$ : $F \times S^{1} \rightarrow G \times S^{1}$. Let

$$
S=(W-n b d(F)) \cup_{\tilde{\phi}}(P-n b d(G))
$$

Then there is a surjection

$$
\pi_{1}(P) \rightarrow \pi_{1}(S)
$$

whose kernel contains the image of $\pi_{1}(F) \rightarrow \pi_{1}(P)$.

q.e.d.

3.2. Our second building block $W_{2}$ is similar to $W_{1}$ but starts with $T^{4}$ instead of $T^{2} \times S^{2}$ :

$$
W_{2}=T^{2} \times T^{2} \# 2 \overline{\mathbb{C P}}^{2} .
$$

We use the calculations of Section 2 to identify two Lagrangian tori $T_{1}$ and $T_{2}$ in $W_{2}$ and calculate the fundamental group of $W_{2}-\left(T_{1} \cup T_{2}\right)$, as well as their meridians and Lagrangian push offs. 
Recall from Section 2 that $\hat{H}, \hat{K}$ are 2-tori, $H$ is the complement of a small disk in $\hat{H}$, and $K$ is the complement of a small disc in $\hat{K}=T^{2}$.

Denote $T^{4} \# 2 \overline{\mathbb{C P}}^{2}=(\hat{H} \times \hat{K}) \# 2 \overline{\mathbb{C P}}^{2}$ by $W_{2}$. Then $W_{2}$ contains a symplectic surface $F_{2}$ of genus 2 with trivial normal bundle. The construction is similar to that of $F_{1} \subset W_{1}$. Start with the symplectic surface $\hat{H} \times\{k\} \cup\{h\} \times \hat{K} \subset T^{4}$. Symplectically resolve the double point to obtain a symplectic surface $F_{2} \subset T^{4}$ of square $([H]+[K])^{2}=2$. Blow up at two points on $F_{2}$ to obtain $W_{2}$ and denote again by $F_{2} \subset W_{2}$ the proper transform.

Notice that $W_{2}-n b d\left(F_{2}\right)$ contains the two Lagrangian tori $T_{1}=$ $X \times A_{1}$ and $T_{2}=Y \times A_{2}$ from Theorem 1. These Lagrangian tori miss the two exceptional spheres, since $\hat{H} \times \hat{K}$ is blown up at points on $F_{2}$, which misses $T_{1}$ and $T_{2}$.

Recall that $\pi_{1}\left(H \times K-\left(T_{1} \cup T_{2}\right),(h, k)\right)$ is generated by four loops, denoted by $x, y, a, b$ in Section 2. The loops $x, y$ lie on $H \times\{k\}$ and form a basis of $\pi_{1}(\hat{H})$ and the loops $a, b$ lie on $\{h\} \times K$ and form a basis of $\pi_{1}(\hat{K})$. Choose a small 4 -ball neighborhood $B_{(h, k)}$ of $(h, k)$. Since $F_{2}$ is constructed by desingularizing $\hat{H} \times\{k\} \cup\{h\} \times \hat{K}$, we may assume that $F_{2}$ coincides with $\hat{H} \times\{k\} \cup\{h\} \times \hat{K}$ outside $B_{(h, k)}$. One can choose loops $s_{1}, t_{1}, s_{2}, t_{2}$ on $F_{2}$ based at point $w$ in $F_{2} \cap B_{(h, k)}$ which form the standard generators of $\pi_{1}\left(F_{2}, w\right)$ (in particular the relation $\left[s_{1}, t_{1}\right]\left[s_{2}, t_{2}\right]=1$ holds) and which coincide with the loops $x, y, a, b$ outside a small ball neighborhood of $(h, k)$.

Proposition 6. The symplectic surface $F_{2} \subset W_{2}$ intersects an embedded sphere transversally in one point. This sphere is disjoint from $T_{1} \cup T_{2}$, and hence the inclusion $W_{2}-n b d\left(F_{2} \cup T_{1} \cup T_{2}\right) \subset W_{2}-n b d\left(T_{1} \cup T_{2}\right)$ induces an isomorphism on fundamental groups, as does $Q-n b d(F) \subset Q$ for any manifold $Q$ obtained by any torus surgeries on $T_{1}$ and $T_{2}$ in $W_{2}$.

The fundamental group $\pi_{1}\left(W_{2}-n b d\left(T_{1} \cup T_{2}\right), w\right)$ is generated by the loops $s_{1}, t_{1}, s_{2}, t_{2}$, which lie on $F_{2}$. The relations

$$
\left[s_{1}, s_{2}\right]=1,\left[t_{1}, s_{2}\right]=1
$$

as well as

$$
\left[s_{1}, t_{1}\right]=1,\left[s_{2}, t_{2}\right]=1
$$

hold in $\pi_{1}\left(W_{2}-n b d\left(T_{1} \cup T_{2}\right), w\right)$.

Moreover, one can choose paths in $W_{2}-n b d\left(T_{1} \cup T_{2}\right)$ from $w$ to the boundary of the tubular neighborhoods of $T_{1}$ and $T_{2}$ so that the meridian and the two Lagrangian push offs of $T_{1}$ are represented in the fundamental group $\pi_{1}\left(W_{2}-n b d\left(T_{1} \cup T_{2}\right), w\right)$ as

$$
\mu_{1}=\left[t_{2}^{-1}, t_{1}^{-1}\right], m_{1}=s_{1}, \ell_{1}=s_{2}
$$

and of $T_{2}$ are

$$
\mu_{2}=\left[s_{1}^{-1}, t_{2}\right], m_{2}=t_{1}, \ell_{2}=t_{2} s_{2} t_{2}^{-1}=s_{2} .
$$


Proof. The assertions all follow from the construction and Theorem 1, except the relations $\left[s_{1}, t_{1}\right]=1$ and $\left[s_{2}, t_{2}\right]=1$. The relation $\left[s_{1}, t_{1}\right]=1$ holds in $W_{2}-\left(T_{1} \cup T_{2}\right)$ because the loops $s_{1}$ and $t_{1}$ agree with the two generators $x, y$ of the fundamental group of the torus $\hat{H} \times\{k\} \subset$ $W_{2}-\left(T_{1} \cup T_{2}\right)$ outside a small 4-ball neighborhood of the base point. The relation $\left[s_{2}, t_{2}\right]=1$ follows from the surface relation $\left[s_{1}, t_{1}\right]\left[s_{2}, t_{2}\right]$, since $F_{2}$ lies in $W_{2}-\left(T_{1} \cup T_{2}\right)$.

q.e.d.

Since $F_{2}$ meets a sphere in $W_{2}-n b d\left(T_{1} \cup T_{2}\right)$ transversally in one point, and $\pi_{1}\left(F_{2}\right) \rightarrow \pi_{1}\left(W_{2}-n b d\left(T_{1} \cup T_{2}\right)\right)$ is surjective, Lemma 4 applies to the pair $\left(W_{2}-n b d\left(T_{1} \cup T_{2}\right), F_{2}\right)$. Thus if $P$ is any manifold containing a genus 2 surface $G$ with trivialized normal bundle, and $\phi: F_{2} \rightarrow G$ is a diffeomorphism, then the sum

$$
S=\left(W_{2}-n b d\left(F_{2}\right)\right) \cup_{\tilde{\phi}}(P-n b d(G))
$$

has fundamental group a quotient of $\pi_{1}(P, \phi(w))$, as does

$$
S-n b d\left(T_{1} \cup T_{2}\right)=\left(W_{2}-n b d\left(F_{2} \cup T_{1} \cup T_{2}\right)\right) \cup_{\tilde{\phi}}(P-n b d(G)) .
$$

Applying Proposition 6 we conclude that

1) The kernel of the surjection $\pi_{1}(P, \phi(w)) \rightarrow \pi_{1}\left(S-n b d\left(T_{1} \cup T_{2}\right), w\right)$ contains the classes

$$
\phi\left(\left[s_{1}, s_{2}\right]\right), \phi\left(\left[t_{1}, s_{2}\right]\right), \phi\left(\left[t_{1}, t_{2} s_{2} t_{2}^{-1}\right]\right), \phi\left(\left[s_{1}, t_{1}\right]\right), \phi\left(\left[s_{2}, t_{2}\right]\right) .
$$

2) The meridians and Lagrangian push offs of $T_{1}$ and $T_{2}$ in $S$ with respect to appropriate paths to the boundary of their tubular neighborhood are given by the images of

$$
\mu_{1}=\phi\left(\left[t_{2}^{-1}, t_{1}^{-1}\right]\right), m_{1}=\phi\left(s_{1}\right), \ell_{1}=\phi\left(s_{2}\right)
$$

and

$$
\mu_{2}=\phi\left(\left[s_{1}^{-1}, t_{2}\right]\right), m_{2}=\phi\left(t_{1}\right), \ell_{2}=\phi\left(s_{2}\right)
$$

under the surjection

$$
\pi_{1}(P, \phi(w)) \rightarrow \pi_{1}\left(S-n b d\left(T_{1} \cup T_{2}\right), w\right)
$$

and hence if $S^{\prime}$ is obtained from $1 / k_{i}$ Luttinger surgery on $T_{i}$ along $\gamma_{i}=m_{i}^{p_{i}} \ell_{i}^{q_{i}}$ for $i=1,2$, then the kernel of the corresponding surjection

$$
\left.\pi_{1}(P, \phi(w)) \rightarrow \pi_{1}\left(S^{\prime}\right)\right)
$$

contains the classes $\phi\left(\left[t_{2}^{-1}, t_{1}^{-1}\right]\left(s_{1}^{p_{1}} s_{2}^{q_{1}}\right)^{k_{1}}\right)$ and $\phi\left(\left[s_{1}^{-1}, t_{2}\right]\left(t_{1}^{p_{2}} s_{2}^{q_{2}}\right)^{k_{2}}\right)$. 
3.3. The final and most complicated building block $M$ is a product $\hat{H} \times \Sigma$ of a torus $\hat{H}$ with a genus 2 surface $\Sigma$. Give $M$ the product symplectic form. We will identify four Lagrangian tori $T_{1}, T_{2}, T_{3}$, and $T_{4}$ and a genus two symplectic surface $F$ in $M$ which are pairwise disjoint and compute the fundamental group of $M-\operatorname{nbd}\left(F \cup \cup_{i=1}^{4} T_{i}\right)$ and all meridians and Lagrangian push offs.

In contrast to $W_{1}$ and $W_{2}, M$ contains no exceptional spheres since $\pi_{2}(M)=0$. In particular the inclusion

$$
M-\operatorname{nbd}\left(F \cup_{i=1}^{4} T_{i}\right) \subset M-\operatorname{nbd}\left(\cup_{i=1}^{4} T_{i}\right)
$$

does not induce an isomorphism on fundamental groups. Thus we will have to be extremely careful when choosing generating loops and computing the fundamental groups of symplectic sums with $M$.

Our approach is to view $M$ as the union of two copies of $\hat{H} \times K$ from Section 2. The main technical difficulty which arises is that of identifying the generators of the fundamental group of the boundary of a tubular neighborhood of $F$ to the generators constructed from Theorem 1. This is critical in order to properly set up the use of the Seifert-Van Kampen theorem.

Let $D$ be a disk with center $u$ in $\hat{H}$ and identify the complement of $D$ with the surface $H$ of Section 2. Thus we have curves $x, y, X, Y$ and the point $h$ in $\hat{H}$ for Figure 1. To each point $q \in \hat{H}$, write

$$
\Sigma_{q}=\{q\} \times \Sigma .
$$

The surface $\Sigma_{u}$ corresponding to the center $u$ of the disk $D$ will play a special role in the following, so that we denote it by $F$ :

$$
F=\{u\} \times \Sigma .
$$

The surfaces $\Sigma_{q}$ are symplectic for all $q$. Moreover, if $q$ misses $X \cup Y$ then $\Sigma_{q}$ misses all the $T_{i}$. Fix $h^{\prime}$ in the boundary of the disk $D$ and choose an $\operatorname{arc} \alpha$ in $\hat{H}$ joining $h^{\prime}$ to $h$, as in Figure 2.

Next view the genus 2 surface $\Sigma$ as the union of two copies of $K$ along their boundary, $\Sigma=K_{1} \cup_{\partial K_{1}=\partial K_{2}} K_{2}$. Thus we have curves $a_{1}, b_{1}$ on $K_{1}$ and $a_{2}, b_{2}$ on $K_{2}$. Choose arcs $\beta_{1}$ (resp. $\beta_{2}$ ) from a point $k^{\prime}$ on the circle separating $K_{1}$ and $K_{2}$ in $\Sigma$ to the intersection point $k_{1}$ of $a_{1}$ and $b_{1}$ (resp. $k_{2}$ of $a_{2}$ and $b_{2}$ ). Use the $\beta_{i}$ to define the corresponding based homotopy classes which satisfy $\left[a_{1}, b_{2}\right]\left[a_{2}, b_{2}\right]=1$ in $\pi_{1}\left(K, k^{\prime}\right)$. Choose two loops $A_{1}, A_{2}$ parallel to $a_{1}$ in $K_{1}$ and $A_{3}, A_{4}$ parallel to $a_{2}$ in $K_{2}$.

The notation is illustrated in Figure 2.

The product $M=\hat{H} \times \Sigma$ contains four disjoint Lagrangian tori $T_{1}=$ $X \times A_{1}, T_{2}=Y \times A_{2}, T_{3}=X \times A_{3}$ and $T_{4}=Y \times A_{4}$ and the symplectic surface $F=\Sigma_{u}$. These five surfaces are pairwise disjoint. 


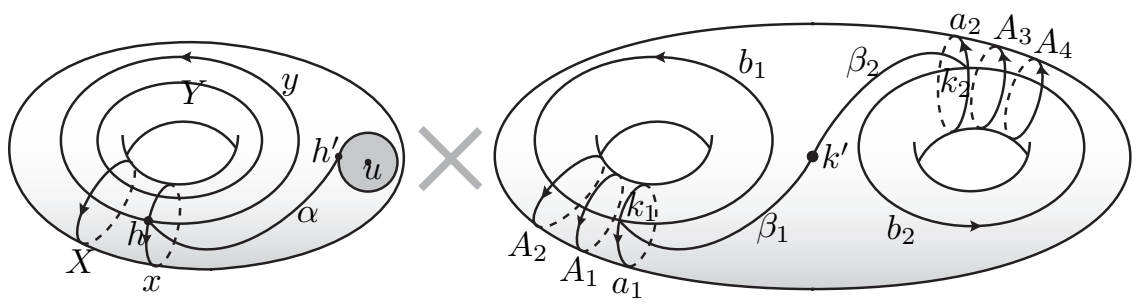

Figure 2. The surface $\hat{H} \times \Sigma$.

The boundary $\partial D \times \Sigma$ of the tubular neighborhood of $F$ in $M$ contains the push off $\Sigma_{h^{\prime}}$ of $F$, as well as a meridian $\mu_{F}=\partial D \times\left\{k^{\prime}\right\}$. We think of $\partial(n b d(F))$ as $\mu_{F} \times \Sigma_{h^{\prime}}$, with base point $\left(h^{\prime}, k^{\prime}\right)$.

The work we do in the rest of this subsection amounts to finding loops on $\Sigma_{h^{\prime}}$ and paths between the different base points to allow us to understand the homomorphism

$$
\pi_{1}(\partial(n b d(F))) \rightarrow \pi_{1}\left(M-n b d\left(F \cup_{i=1}^{4} T_{i}\right)\right)
$$

explicitly.

For convenience denote by $N$ the open tubular neighborhood in $M$ of the union of $F$ and the Lagrangian tori:

$$
N=(D \times \Sigma) \cup n b d\left(T_{1} \cup T_{2} \cup T_{3} \cup T_{4}\right) .
$$

Give $M$ the base point $p=\left(h^{\prime}, k^{\prime}\right)$ on the boundary of the tubular neighborhood of $F$. We define six loops in $M-N$ based at $p$.

1) The loop $x \times\left\{k^{\prime}\right\}$ lies on $H \times\left\{k^{\prime}\right\} \subset M-N$ and is based at $\left(h, k^{\prime}\right)$. We conjugate this by the path $\alpha \times\left\{k^{\prime}\right\}$ to define a loop $\tilde{x}$ based at $p=\left(h^{\prime}, k^{\prime}\right)$ :

$$
\tilde{x}=\left(\alpha * x * \alpha^{-1}\right) \times\left\{k^{\prime}\right\}: I \rightarrow H \times\left\{k^{\prime}\right\} \subset M-N .
$$

2) The loop $y \times\left\{k^{\prime}\right\}$ lies on $H \times\left\{k^{\prime}\right\} \subset M-N$ and is based at $\left(h, k^{\prime}\right)$. We conjugate this by the path $\alpha \times\left\{k^{\prime}\right\}$ to define a loop $\tilde{y}$ based at $p$ :

$$
\tilde{y}=\left(\alpha * y * \alpha^{-1}\right) \times\left\{k^{\prime}\right\}: I \rightarrow H \times\left\{k^{\prime}\right\} \subset M-N .
$$

3) The loops $a_{1}, b_{1}, a_{2}, b_{2}$ on $\Sigma$ defined above determine loops on $\Sigma_{h^{\prime}} \subset \partial(D \times \Sigma)$ based at $p$ :

$$
\begin{aligned}
& \tilde{a}_{1}=\left\{h^{\prime}\right\} \times a_{1} \\
& \tilde{b}_{1}=\left\{h^{\prime}\right\} \times b_{1} \\
& \tilde{a}_{2}=\left\{h^{\prime}\right\} \times a_{2} \\
& \tilde{b}_{2}=\left\{h^{\prime}\right\} \times b_{2} .
\end{aligned}
$$


Thus the loops $\tilde{a}_{1}, \tilde{b}_{1}, \tilde{a}_{2}$, and $\tilde{b}_{2}$ lie on the push off $\Sigma_{h^{\prime}}$ of $F$ in the boundary of the tubular neighborhood of $F$. Together with the loop $\mu_{F}=\partial D \times\left\{k^{\prime}\right\}$, they generate the fundamental group of $\partial(n b d(F))=$ $\partial D \times \Sigma_{h^{\prime}}$ based at $p$.

By contrast, away from the base point, the loops $\tilde{x}$ and $\tilde{y}$ lie in the interior of $M-N$. However, their commutator $[\tilde{x}, \tilde{y}]$ equals $\mu_{F}$ in the group $\pi_{1}(M-N, p)$, since the punctured torus $H \times\left\{k^{\prime}\right\} \subset M-N$ has boundary $\mu_{F}$.

At first glance, the following proposition may appear to be a direct application of the Seifert-Van Kampen applied to two copies of the manifold of Theorem 1. However, the base point in Theorem 1 does not lie on the boundary. Thus we must change base point and homotope appropriate loops into the boundary of $M-N$, being careful not to homotope the loops through $N$ in the process.

Proposition 7. The fundamental group $\pi_{1}\left(M-n b d\left(F \cup_{i=1}^{4} T_{i}\right), p\right)$ is generated by $\tilde{x}, \tilde{y}, \tilde{a}_{1}, \tilde{b}_{1}, \tilde{a}_{2}, \tilde{b}_{2}$ and the relations

$$
1=\left[\tilde{x}, \tilde{a}_{1}\right]=\left[\tilde{y}, \tilde{a}_{1}\right]=\left[\tilde{y}, \tilde{b}_{1} \tilde{a}_{1} \tilde{b}_{1}^{-1}\right]=\left[\tilde{x}, \tilde{a}_{2}\right]=\left[\tilde{y}, \tilde{a}_{2}\right]=\left[\tilde{y}, \tilde{b}_{2} \tilde{a}_{2} \tilde{b}_{2}^{-1}\right]
$$

hold in this group. With respect to certain paths to the boundary of the tubular neighborhoods of the $T_{i}$, the meridian and two Lagrangian push offs are given by

1) $T_{1}: \mu_{1}=\left[\tilde{b}_{1}^{-1}, \tilde{y}^{-1}\right], m_{1}=\tilde{x}, \ell_{1}=\tilde{a}_{1}$,

2) $T_{2}: \mu_{2}=\left[\tilde{x}^{-1}, \tilde{b}_{1}\right], m_{2}=\tilde{y}, \ell_{2}=\tilde{b}_{1} \tilde{a}_{1} \tilde{b}_{1}^{-1}$,

3) $T_{3}: \mu_{3}=\left[\tilde{b}_{2}^{-1}, \tilde{y}^{-1}\right], m_{3}=\tilde{x}, \ell_{3}=\tilde{a}_{2}$,

4) $T_{4}: \mu_{4}=\left[\tilde{x}^{-1}, \tilde{b}_{2}\right], m_{4}=\tilde{y}, \ell_{4}=\tilde{b}_{2} \tilde{a}_{2} \tilde{b}_{2}^{-1}$.

The loops $\tilde{a}_{1}, \tilde{b}_{1}, \tilde{a}_{2}, \tilde{b}_{2}$ lie on the genus 2 surface $\Sigma_{h^{\prime}}$ and form a standard set of generators (so $\left[\tilde{a}_{1}, \tilde{b}_{1}\right]\left[\tilde{a}_{2}, \tilde{b}_{2}\right]=1$ ). These four loops and a meridian $\mu_{F}$ generate the fundamental group of the boundary of the tubular neighborhood of $F$, and $\mu_{F}$ is homotopic to $[\tilde{x}, \tilde{y}]$ in the group $\pi_{1}\left(M-n b d\left(F \cup_{i=1}^{4} T_{i}\right), p\right)$.

(Please see the remark which follows the proof.)

Proof. First notice that the punctured torus $H \times\left\{k^{\prime}\right\}$ misses the tubular neighborhood $N$. Since the path $\alpha \times\left\{k^{\prime}\right\}$ lies in $H \times\left\{k^{\prime}\right\}$, the boundary of this punctured torus represents the same based homotopy class as $[\tilde{x}, \tilde{y}]$ in $\pi_{1}(M-N, p)$. This represents the meridian $\mu_{F}$.

The boundary of the tubular neighborhood of $F$ is trivialized by the push off $\Sigma_{h^{\prime}}$. The curves $\tilde{a}_{1}, \tilde{b}_{1}, \tilde{a}_{2}, \tilde{b}_{2}$ lie on this push off and so these four loops and $\mu_{F}$ generate the fundamental group of the boundary of the tubular neighborhood of $F$, based at $p$.

Let $S \subset \Sigma$ denote the circle separating $\Sigma$ into the two punctured tori $K_{1}$ and $K_{2}$. Cutting $M-n b d(F)$ along $H \times S$ exhibits $M-n b d(F)$ as the union of two copies of $H \times K$, where $H$ and $K$ are punctured tori. 
The first copy $H_{1} \times K_{1}$ contains the two Lagrangian tori $T_{1}$ and $T_{2}$ and the other contains the tori $T_{3}$ and $T_{4}$.

After cutting $M-n b d(F)$, the surface $H \times\left\{k^{\prime}\right\}$ appears as the codimension 1 submanifold $H_{1} \times\left\{k_{1}^{\prime}\right\}$ of $\partial\left(H_{1} \times K_{1}\right)$ and as the submanifold $H_{2} \times\left\{k_{2}^{\prime}\right\}$ of $\partial\left(H_{2} \times K_{2}\right)$. Call the copies of $\tilde{x}$ and $\tilde{y}$ that appear in $H_{1} \times\left\{k_{1}^{\prime}\right\} \tilde{x}_{1}$ and $\tilde{y}_{1}$, and in the other component $\tilde{x}_{2}$ and $\tilde{y}_{2}$. The copy of $\left\{h^{\prime}\right\} \times S$ (oriented and based) in $H_{1} \times K_{1}$ represents $\left[\tilde{a}_{1}, \tilde{b}_{1}\right]$ and in $H_{2} \times K_{2}$ represents $\left[\tilde{a}_{2}, \tilde{b}_{2}\right]^{-1}$.

The Seifert-Van Kampen theorem shows that the fundamental group $\pi_{1}\left(M-n b d\left(F \cup_{i=1}^{4} T_{i}\right), p\right)$ is the quotient of the free product

$$
\pi_{1}\left(H_{1} \times K_{1}-\left(T_{1} \cup T_{2}\right),\left(h_{1}^{\prime}, k_{1}^{\prime}\right)\right) * \pi_{1}\left(H_{2} \times K_{2}-\left(T_{3} \cup T_{4}\right),\left(h_{2}^{\prime}, k_{2}^{\prime}\right)\right)
$$

by the normal subgroup generated by $\tilde{x}_{1} \tilde{x}_{2}^{-1}, \tilde{y}_{1} \tilde{y}_{2}^{-1}$ and $\left[\tilde{a}_{1}, \tilde{b}_{1}\right]\left[\tilde{a}_{2}, \tilde{b}_{2}\right]$. In particular, the loops $\tilde{x}, \tilde{y}, \tilde{a}_{1}, \tilde{b}_{1}, \tilde{a}_{2}, \tilde{b}_{2}$ generate the fundamental group $\pi_{1}\left(M-n b d\left(F \cup_{i=1}^{4} T_{i}\right), p\right)$.

We reduce the proof to Theorem 1 by working one side at a time, and so, to ease eye strain, we drop the subscripts 1,2 . Here is what is to be shown: We have loops $\tilde{x}, \tilde{y}, \tilde{a}, \tilde{b}$ in $H \times K-\left(T_{1} \cup T_{2}\right)$ based at $\left(h^{\prime}, k^{\prime}\right)$ defined earlier in this section, and loops $x, y, a, b$ based at $(h, k)$ defined in the paragraph preceding the statement of Theorem 1 . The loops $x, y, a, b$ satisfy the conclusions which we will show the $\tilde{x}, \tilde{y}, \tilde{a}, \tilde{b}$ satisfy.

We first move from $p=\left(h^{\prime}, k^{\prime}\right)$ to $\left(h, k^{\prime}\right)$. Recall we have the path $\alpha$ from $h^{\prime}$ to $h$ in $H$. We let $\tilde{\alpha}$ denote the path $\alpha \times\left\{k^{\prime}\right\}$. Then conjugation by the path $\tilde{\alpha}^{-1}$ defines an isomorphism

$$
\begin{gathered}
\Psi_{1}: \pi_{1}\left(H \times K-\left(T_{1} \cup T_{2}\right), p\right) \rightarrow \pi_{1}\left(H \times K-\left(T_{1} \cup T_{2}\right),\left(h, k^{\prime}\right)\right), \\
\Psi_{1}(\gamma)=\tilde{\alpha}^{-1} * \gamma * \tilde{\alpha} .
\end{gathered}
$$

From the definition preceding the statement of Proposition 7 we see that $\Psi_{1}(\tilde{x})$ and $\Psi_{1}(\tilde{y})$ are homotopic rel $\left(h, k^{\prime}\right)$ to the loops $x \times\left\{k^{\prime}\right\}$ and $y \times\left\{k^{\prime}\right\}$, since e.g.

$$
\Psi_{1}(\tilde{x})=\tilde{\alpha}^{-1} * \tilde{x} * \tilde{\alpha}=\tilde{\alpha}^{-1} \tilde{\alpha} *\left(x \times\left\{k^{\prime}\right\}\right) * \tilde{\alpha}^{-1} \tilde{\alpha} \sim x \times\left\{k^{\prime}\right\} .
$$

Recall that $\tilde{a}$ takes the form $\left\{h^{\prime}\right\} \times\left(\beta * a * \beta^{-1}\right)$, where $\beta$ is the given path in $K$ from $k^{\prime}$ to $k$, and similarly for $\tilde{b}$.

The free homotopy $t \mapsto\{\alpha(t)\} \times\left(\beta * a * \beta^{-1}\right)$ from $\tilde{a}$ to $\{h\} \times(\beta *$ $a * \beta^{-1}$ ) misses $T_{1} \cup T_{2}$ and drags the base point along $\tilde{\alpha}$. Hence $\Psi_{1}(\tilde{a})$ is represented by the loop $\{h\} \times\left(\beta * a * \beta^{-1}\right)$ which lies on $\{h\} \times K$. Similarly $\Psi_{1}(\tilde{b})$ is represented by the loop $\{h\} \times\left(\beta * b * \beta^{-1}\right)$.

Now we use conjugation by the path $\tilde{\beta}=\{h\} \times \beta$ to define an isomorphism

$$
\Psi_{2}: \pi_{1}\left(H \times K-\left(T_{1} \cup T_{2}\right),\left(h, k^{\prime}\right)\right) \rightarrow \pi_{1}\left(H \times K-\left(T_{1} \cup T_{2}\right),(h, k)\right)
$$




$$
\Psi_{2}(\gamma)=\tilde{\beta}^{-1} * \gamma * \tilde{\beta} .
$$

This takes the loop $\Psi_{1}(\tilde{a})=\{h\} \times\left(\beta * a * \beta^{-1}\right)$ to $\{h\} \times a$ :

$$
\Psi_{2}\left(\Psi_{1}(\tilde{a})\right)=\tilde{\beta}^{-1} *\left(\{h\} \times\left(\beta * a * \beta^{-1}\right)\right) * \tilde{\beta} \sim\{h\} \times a .
$$

Similarly $\Psi_{2}\left(\Psi_{1}(\tilde{b})\right)=\{h\} \times b$. These are the loops simply denoted by $a$ and $b$ in Theorem 1 .

The free homotopy $t \mapsto x \times\{\beta(t)\}$ starts at $x \times\left\{k^{\prime}\right\}=\Psi_{1}(\tilde{x})$ and ends at $x \times\{k\}$, which is the loop labeled by $x$ in Theorem 1. Moreover, the loop $x \times\{\beta(t)\}$ misses $T_{1} \cup T_{2}$, since $\beta$ avoids $A_{1}$ and $A_{2}$. Since this free homotopy drags the base point along $\{h\} \times \beta=\tilde{\beta}$, it shows that $\Psi_{2}\left(\Psi_{1}(\tilde{x})\right) \sim x \times\{k\}$. Similarly $\Psi_{2}\left(\Psi_{1}(\tilde{y})\right) \sim y \times\{k\}$.

Thus we have found a path $\tau=\tilde{\beta} * \tilde{\alpha}$ in $H \times K-\left(T_{1} \cup T_{2}\right)$ from $(h, k)$ to $\left(h^{\prime}, k^{\prime}\right)$ and proven that the isomorphism

$$
\pi_{1}\left(H \times K-\left(T_{1} \cup T_{2}\right),\left(h^{\prime}, k^{\prime}\right)\right) \rightarrow \pi_{1}\left(H \times K-\left(T_{1} \cup T_{2}\right),(h, k)\right)
$$

given by conjugating by $\tau^{-1}$ takes (the based homotopy classes of) $\tilde{x}, \tilde{y}, \tilde{a}, \tilde{b}$ to (the based homotopy classes of) $x, y, a, b$. Hence any relation satisfied by $x, y, a, b$ in $\pi_{1}\left(H \times K-\left(T_{1} \cup T_{2}\right),(h, k)\right)$ is also satisfied by $\tilde{x}, \tilde{y}, \tilde{a}, \tilde{b}$ in $\pi_{1}\left(H \times K-\left(T_{1} \cup T_{2}\right),\left(h^{\prime}, k^{\prime}\right)\right)$.

Moreover, if one takes the paths from $\left(h^{\prime}, k^{\prime}\right)$ to the boundary of the tubular neighborhood of $T_{i}$ to be the composite of $\tau$ and the path given in Theorem 1, then e.g. the meridian of $T_{1}$ with respect to this path is

$$
\tau * \mu_{1} * \tau^{-1}=\left(\Psi_{2} \circ \Psi_{1}\right)^{-1}\left(\mu_{1}\right)=\left(\Psi_{2} \circ \Psi_{1}\right)^{-1}\left(\left[b^{-1}, y^{-1}\right]\right)=\left[\tilde{b}^{-1}, \tilde{y}^{-1}\right] .
$$

A similar argument establishes the calculations for the other meridian and the Lagrangian push offs.

Applying the argument on each half $H_{i} \times K_{i} i=1,2$ and using the Seifert-Van Kampen theorem finishes the proof. q.e.d.

Remark. To simplify notation, for the rest of this paper we drop the decorations, and so we will denote $\tilde{x}$ simply by $x$ and similarly for the others. Thus the explicit loops in $M-N$ based at $p=\left(h^{\prime}, k^{\prime}\right)$ defined prior to Proposition 7 will be denoted by $x, y, a_{1}, b_{1}, a_{2}, b_{2} . a_{1}, b_{1}, a_{2}, b_{2}$ are loops that lie on $\Sigma_{h^{\prime}}$ and together with $\mu_{F}$ generate the fundamental group of the boundary of the tubular neighborhood of $F$.

The loops $x, y$ lie on the surface $H \times\left\{k^{\prime}\right\}$ (and in particular in the interior of $M-N$ away from $p$ ). The meridian $\mu_{F}$ equals $[x, y]$ in $\pi_{1}(M-N, p)$, and the loops $x, y, a_{1}, b_{1}, a_{2}, b_{2}$ generate $\pi_{1}(M-N, p)$, with relations, meridians, and Lagrangian push offs as given in Proposition 7 . 


\section{Constructions of small simply symplectic manifolds}

4.1. We start, as a warm up, with a construction of a minimal symplectic manifold homeomorphic but not diffeomorphic to $\mathbb{C P}^{2} \# 7 \overline{\mathbb{C P}}^{2}$. Such examples are known $[\mathbf{2 7}, \mathbf{2 5}]$; we include it because our construction illustrates the kind of fundamental group calculations we will do below in a simple case.

Theorem 8. One can perform two Luttinger surgeries on the symplectic sum of $W_{1}$ and $W_{2}$ along $F_{1}$ and $F_{2}$ to produce a minimal symplectic manifold $U$ homeomorphic but not diffeomorphic to $\mathbb{C P}^{2} \# 7 \overline{\mathbb{C P}}^{2}$.

Proof. Form the symplectic sum $S=W_{1}-n b d\left(F_{1}\right) \cup_{\tilde{\phi}} W_{2}-n b d\left(F_{2}\right)$ using the gluing diffeomorphism $\phi: F_{1} \rightarrow F_{2}$ which take the loops denoted by $s_{1}, t_{1}, s_{2}, t_{2}$ on $F_{1}$ to their namesakes on $F_{2}$. The Lagrangian tori $T_{1}, T_{2}$ in $W_{2}$ remain Lagrangian in $S$ ([16, Theorem 10.2.1]).

Lemma 4 shows that $\pi_{1}\left(S-\left(T_{1} \cup T_{2}\right)\right)$ is a quotient of the group $\pi_{1}\left(W_{2}-\left(T_{1} \cup T_{2}\right)\right)$ and the kernel of the surjection contains the classes $s_{1} s_{2}, t_{1} t_{2}$, and $\left[s_{1}, t_{1}\right]$. Applying Proposition 6 we see that the group $\pi_{1}\left(S-\left(T_{1} \cup T_{2}\right)\right)$ is a quotient of the group generated by $s_{1}, t_{1}$ and the relation $\left[s_{1}, t_{1}\right]=1$ holds, i.e. $\pi_{1}\left(S-\left(T_{1} \cup T_{2}\right)\right)$ is a quotient of $\mathbb{Z} s_{1} \oplus \mathbb{Z} t_{1}$. Moreover, the meridians and Lagrangian push offs of the tori $T_{i}$ are given by

$$
\mu_{1}=1, m_{1}=s_{1}, \ell_{1}=s_{1}^{-1}
$$

and

$$
\mu_{2}=1, m_{2}=t_{1}, \ell_{2}=s_{1}^{-1} .
$$

We perform Luttinger surgeries on $T_{1}$ and $T_{2}$ in $S$ or, equivalently, Luttinger fillings on $S-n b d\left(T_{1} \cup T_{2}\right)$. Then $-1 / 1$ Luttinger surgery on $T_{1}$ along $m_{1}$ kills $s_{1}$ and $-1 / 1$ Luttinger surgery on $T_{2}$ along $m_{2}$ then kills $t_{1}$, yielding a simply connected symplectic manifold $U$. Notice that Luttinger surgery does not change the Euler characteristic nor the signature of a 4-manifold. Thus we have

$$
e(U)=e\left(W_{1}\right)+e\left(W_{2}\right)+4=10
$$

and, using Novikov additivity,

$$
\sigma(U)=\sigma\left(W_{1}-n b d\left(F_{1}\right)\right)+\sigma\left(W_{2}-n b d\left(F_{2}\right)\right)=\sigma\left(W_{1}\right)+\sigma\left(W_{2}\right)=-6 .
$$

Freedman's theorem $[\mathbf{1 4}]$ then implies that the 4-manifold $U$ is homeomorphic to $\mathbb{C P}^{2} \# 7 \overline{\mathbb{C P}}^{2}$.

We showed that every -1 sphere in $W_{1}$ meets $F_{1}$ in Proposition 3 . Let $W_{2}^{\prime}$ be the manifold obtained from $W_{2}$ by performing the Luttinger surgeries as described, so that $U$ is the symplectic sum of $W_{1}$ and $W_{2}^{\prime}$. To see that every -1 sphere in $W_{2}^{\prime}$ intersects $F_{2}$ takes a bit more work. Notice that $W_{2}^{\prime}$ is obtained by performing the two Luttinger surgeries on $T_{1}$ and $T_{2}$ in $T^{4}=\hat{H} \times \hat{K}$ and then blowing up twice along $F_{2}$. 
These are Luttinger surgeries along $m_{1}$ and $m_{2}$, hence by Lemma $2, W_{2}^{\prime}$ is obtained by blowing up an aspherical manifold $L$ twice along points on $F_{2}$.

Since $L$ is aspherical and $\pi_{1}\left(W_{2}^{\prime}\right) \rightarrow \pi_{1}(L)$ is an isomorphism, the Hopf sequence (see [9, pg. 265])

$$
\pi_{2}\left(W_{2}^{\prime}\right) \rightarrow H_{2}\left(W_{2}^{\prime}\right) \rightarrow H_{2}(L) \rightarrow 0
$$

is exact, where $W_{2}^{\prime} \rightarrow L$ is the map that collapses the two exceptional spheres (i.e. the blow-down map). The kernel of $H_{2}\left(W_{2}^{\prime}\right) \rightarrow H_{2}(L)$ is clearly generated by the two exceptional spheres $E_{1}$ and $E_{2}$, and therefore every spherical class in $H_{2}\left(W_{2}^{\prime}\right)$ has the form $a E_{1}+b E_{2}$. In particular, the only -1 spheres are $\pm E_{1}$ and $\pm E_{2}$, and both of these intersect $F$.

If $W_{1}$ were an $S^{2}$ bundle with section $F_{1}$, then $\pi_{1}\left(W_{1}\right)$ could not be $\mathbb{Z}^{2}$. If $W_{2}^{\prime}$ were an $S^{2}$ bundle with section $F_{2}$, then the exact sequence in homotopy groups of a fibration would show that $\pi_{2}\left(W_{2}^{\prime}\right)$ equals $\pi_{2}\left(S^{2}\right)=$ $\mathbb{Z}$. But we showed in the previous paragraph that the rank of the image of the Hurewicz map equals 2. (One can also compute directly that $H_{1}\left(W_{2}^{\prime}\right)$ is generated by $s_{2}$ and $t_{2}$, so that $\pi_{1}\left(W_{2}^{\prime}\right) \approx \pi_{1}\left(F_{2}\right)$.) Thus $W_{2}^{\prime}$ cannot be an $S^{2}$ bundle over $F_{2}$. Applying Usher's theorem [39], we conclude that $U$ is a minimal symplectic 4-manifold.

By results of Taubes, [38, Theorem 0.2], a minimal symplectic 4manifold cannot contain a smoothly embedded -1 sphere, but the manifold $\mathbb{C P}^{2} \# 7 \overline{\mathbb{C P}}^{2}$ contains smoothly embedded -1 spheres, namely, the exceptional spheres. Hence $U$ cannot be diffeomorphic to $\mathbb{C P}^{2} \# 7 \overline{\mathbb{C P}}^{2}$, since this would contradict the minimality of $U$.

q.e.d.

4.2. Our next example is more involved. We take a symplectic sum of $W_{1}$ and $M$ and perform four Luttinger surgeries to produce a minimal symplectic manifold homeomorphic but not diffeomorphic to $\mathbb{C P}^{2} \# 5 \overline{\mathbb{C P}}^{2}$ (cf. $[29,2]$ ).

Consider the surface $H$ and its curves $x, y, X, Y$ and base point $h$ in Figure 1. Let $D_{X}$ and $D_{Y}$ denote the Dehn twists along $X$ and $Y$. We leave the proof of the following simple lemma to the reader.

Lemma 9. The composite of Dehn twists along $X$ and $Y$ given by $D_{X} D_{Y} D_{X}$ takes $x$ to $y^{-1}$ and $y$ to $y x y^{-1}$ in $\pi_{1}(H, h)$ q.e.d.

Note Lemma 9 holds in $\pi_{1}\left(H, h^{\prime}\right)$ if we replace $x, y$ by $\alpha * x * \alpha^{-1}$, $\alpha * y * \alpha^{-1}$ where $\alpha$ is the path in $H$ from the boundary point $h^{\prime}$ to $h$ which misses $X$ and $Y$. Moreover, $D_{X} D_{Y} D_{X}$ is the identity on the boundary, so that Lemma 9 extends to higher genus surfaces.

Consider the surface $F_{1} \subset W_{1}$ with base point $w$ and $\Sigma_{h^{\prime}} \subset M$ with base point $p=\left(h^{\prime}, k^{\prime}\right)$. Denote by $\phi: F_{1} \rightarrow \Sigma_{h^{\prime}}$ the base point preserving diffeomorphism obtained by composing the diffeomorphism 
of Lemma 9 (applied to $H=F_{1}$ ) with a diffeomorphism $F_{1} \rightarrow \Sigma_{h^{\prime}}$ taking $s_{i}$ to $a_{i}$ and $t_{i}$ to $b_{i}$. This induces the isomorphism on fundamental groups

$$
\phi: \pi_{1}\left(F_{1}, w\right) \rightarrow \pi_{1}\left(\Sigma_{h^{\prime}}, p\right),\left(s_{1}, t_{1}, s_{2}, t_{2}\right) \mapsto\left(b_{1}^{-1}, b_{1} a_{1} b_{1}^{-1}, a_{2}, b_{2}\right)
$$

and extends to $\tilde{\phi}: F_{1} \times S^{1} \rightarrow \Sigma_{h^{\prime}} \times S^{1}$. The symplectic sum

$$
V^{\prime}=\left(W_{1}-n b d\left(F_{1}\right)\right) \cup\left(M-n b d\left(\Sigma_{h^{\prime}}\right)\right)
$$

contains the four Lagrangian tori $T_{1}, T_{2}, T_{3}, T_{4}$ and a symplectic surface $F$ so that these five surfaces are pairwise disjoint.

Using Lemma 4 and the facts that $s_{1}=s_{2}^{-1}, t_{1}=t_{2}^{-1}$, and $\left[s_{1}, t_{1}\right]$ in $\pi_{1}\left(W_{1}\right)$, we see that $b_{1}=a_{2}$ in $\pi_{1}\left(V^{\prime}-n b d\left(F \cup \cup_{i=1}^{4} T_{i}\right), w\right)$. The relation $\left[s_{1}, t_{1}\right]=1$ implies that $1=\left[b_{1}^{-1}, b_{1} a_{1} b_{1}^{-1}\right]=\left[a_{1}, b_{1}^{-1}\right]$ and hence $\left[a_{1}, a_{2}\right]=\left[a_{1}, b_{1}\right]=1$ and $b_{2}=a_{1}^{-1}$ hold in $\pi_{1}\left(V^{\prime}-n b d\left(F \cup_{i=1}^{4} T_{i}\right), w\right)$.

Therefore, $\pi_{1}\left(V^{\prime}-n b d\left(F \cup_{i=1}^{4} T_{i}\right), w\right)$ is generated by $x, y, a_{1}, a_{2}$ and the relation

$$
\left[a_{1}, a_{2}\right]=1
$$

holds. The relations

$$
\left[x, a_{1}\right]=1,\left[y, a_{1}\right]=1,\left[x, a_{2}\right]=1,\left[y, a_{2}\right]=1
$$

coming from $M-n b d\left(F \cup_{i=1}^{4} T_{i}\right)$ were established in Proposition 7 , and by the Seifert-Van Kampen theorem also hold in $\pi_{1}\left(V^{\prime}-n b d\left(F \cup_{i=1}^{4} T_{i}\right)\right)$.

Furthermore, it follows from Proposition 7 that the meridian of $F$ is

$$
\mu_{F}=[x, y] \text {. }
$$

and the meridians and Lagrangian push offs of the tori $T_{1}$ are given by

- $T_{1}: \mu_{1}=\left[a_{2}^{-1}, y^{-1}\right]=1, m_{1}=x, \ell_{1}=a_{1}$.

- $T_{2}: \mu_{2}=\left[x^{-1}, a_{2}\right]=1, m_{2}=y, \ell_{2}=a_{2} a_{1} a_{2}^{-1}=a_{1}$.

- $T_{3}: \mu_{3}=\left[a_{1}, y^{-1}\right]=1, m_{3}=x, \ell_{3}=a_{2}$.

- $T_{4}: \mu_{4}=\left[x^{-1}, a_{1}^{-1}\right]=1, m_{4}=y, \ell_{4}=a_{1}^{-1} a_{2} a_{1}=a_{2}$.

Theorem 10. There exists a minimal symplectic manifold $V$ homeomorphic but not diffeomorphic to $\mathbb{C P}^{2} \# 5 \overline{\mathbb{C P}}^{2}$ containing a symplectic genus 2 surface $F$ with simply connected complement and trivial normal bundle.

Proof. We do four Luttinger surgeries on $T_{1}, T_{2}, T_{3}$, and $T_{4}$ in $V^{\prime}-F$. First, $-1 / 1$ Luttinger surgery on $T_{1}$ along $m_{1}$ kills $x$. Then $-1 / 1$ surgery on $T_{2}$ along $\ell_{2}$ kills $a_{1}$. Next, $-1 / 1$ surgery on $T_{3}$ along $\ell_{3}$ kills $a_{2}$. Finally, $-1 / 1$ surgery on $T_{4}$ along $m_{4}$ kills $y$.

Thus we have produced a symplectic 4-manifold $V$ such that $V-F$ is simply connected. The Seifert-Van Kampen theorem shows that $V$ is simply connected. The Euler characteristic and signature of $V$ are the same as $V^{\prime}$ since they are unchanged by Luttinger surgery. Hence

$$
e(V)=e\left(W_{1}\right)+e(M)+4=4+0+4=8
$$


and

$$
\sigma(V)=\sigma\left(W_{1}\right)+\sigma(M)=-4+0=-4 .
$$

Since $V$ has an indefinite, odd intersection form (its signature is not a multiple of 8), Freedman's theorem [14] then implies that $V$ is homeomorphic to $\mathbb{C P}^{2} \# 5 \overline{\mathbb{C P}}^{2}$.

Let $M^{\prime}$ be the manifold obtained from $M=\hat{H} \times \Sigma$ by doing the four Luttinger surgeries described above. Minimality of $V$ follows from Usher's theorem [39] just as in the proof of Theorem 8 once we show that $M^{\prime}$ is minimal, and not an $S^{2}$ bundle with section $\Sigma_{h^{\prime}}$. Note that $M^{\prime}$ is the symplectic sum of two manifolds $M_{1}^{\prime}$ and $M_{2}^{\prime}$ along the torus $\hat{H}$.

Indeed, view $M=\hat{H} \times \Sigma$ as the union of $\hat{H}_{1} \times K_{1}$ and $\hat{H}_{2} \times K_{2}$ along $\hat{H} \times S$ as in the proof of Proposition 7. This exhibits $M$ as the symplectic sum of $\hat{H}_{1} \times \hat{K}_{1}$ and $\hat{H}_{2} \times \hat{K}_{2}$ along the symplectic surfaces $\hat{H}_{1} \times\left\{v_{1}\right\}$ and $\hat{H}_{2} \times\left\{v_{2}\right\}$, where $v \in \hat{K}$ is the center of the disk whose complement is $K$. So let $M_{1}^{\prime}$ denote the manifold obtained by Luttinger surgery on $T_{1}$ and $T_{2}$ in the 4-torus $\hat{H}_{1} \times \hat{K}_{1}$ and $M_{2}^{\prime}$ denote the manifold obtained by Luttinger surgery on $T_{3}$ and $T_{4}$ in the 4 -torus $\hat{H}_{2} \times \hat{K}_{2}$.

Lemma 2 implies that $M_{1}^{\prime}$ and $M_{2}^{\prime}$ are aspherical, hence minimal. In $H_{1}\left(M_{1}^{\prime}\right)$, the generators $x$ and $a_{1}$ are zero, so that $H_{1}\left(M_{1}^{\prime}\right)$ is generated by $y$ and $b_{1}$. In particular, $\hat{H}_{1} \times\left\{v_{1}\right\} \subset M_{1}^{\prime}$ cannot be a section of an $S^{2}$ fiber bundle structure on $M_{1}^{\prime}$ since $\pi_{1}\left(\hat{H}_{1} \times\left\{v_{1}\right\}\right)$ is generated by $x$ and $y$. Thus Usher's theorem implies that $M^{\prime}$ is minimal. Finally, $M^{\prime}$ is not an $S^{2}$ bundle with section $\Sigma_{h^{\prime}}$ for similar reasons: the loops $a_{1}, b_{1}, a_{2}, b_{2}$ are generators of $\pi_{1}\left(\Sigma_{h^{\prime}}\right)$ but $a_{1}$ and $a_{2}$ are trivial in $H_{1}\left(M^{\prime}\right)$, as one can readily check. As explained above, it follows that $V$ is minimal.

Using the same argument as in the proof of Theorem 8 one concludes from Taubes's results that $V$ is not diffeomorphic to $\mathbb{C P}^{2} \# 5 \overline{\mathbb{C P}}^{2}$. q.e.d.

Since $V$ contains an appropriate Lagrangian torus, the argument of Corollary 14 below applies to the manifold $V$ as well to produce infinitely many smooth (but not symplectic) pairwise non-diffeomorphic manifolds homeomorphic to $V$.

4.3. We next put together the manifolds $W_{2}$ and $M$. The construction is based on the example of a minimal symplectic manifold homeomorphic to $\mathbb{C P}^{2} \# 3 \overline{\mathbb{C P}}^{2}$ which we constructed in $[6]$. However, the extra information obtained by keeping track of the surface $F$ will allow us to produce many more examples which we will use in constructing small non-simply connected symplectic 4-manifolds in the next section.

Recall that the manifold $W_{2}$ contains a genus 2 square zero symplectic surface $F_{1}$ and two Lagrangian tori $T_{1}$ and $T_{2}$.

The manifold $M=\hat{H} \times \Sigma$ of Proposition 7 contains a symplectic surface $F$ with trivial normal bundle and four Lagrangian tori $T_{1}, T_{2}, T_{3}, T_{4}$, 
with the fundamental group of $M-n b d\left(F \cup T_{1} \cup T_{2} \cup T_{3} \cup T_{4}\right)$ generated by the loops $x, y, a_{1}, b_{1}, a_{2}, b_{2}$ based at $p$ satisfying all the conclusions of Proposition 7.

To avoid notational confusion, we denote the two Lagrangian tori in $W_{2}$ by $T_{1}^{\prime}$ and $T_{2}^{\prime}$. We preserve the notation $T_{1}, T_{2}, T_{3}, T_{4}$ for the four Lagrangian tori in $M$.

The parallel (and symplectic) push off $\Sigma_{h^{\prime}}$ of $F$ lies in the boundary of the tubular neighborhood $D \times \Sigma$ of $F$ and carries the base point and the loops $a_{1}, b_{1}, a_{2}, b_{2}$. We have a framing of $\Sigma_{h^{\prime}} \subset M$ defined by taking a nearby push off $\Sigma_{z}$ for a point $z$ near $h^{\prime}$. Choose some identification of the tubular neighborhood of $F_{2} \subset W_{2}$ with $F_{2} \times D^{2}$.

We form the symplectic sum $Z$ of $W_{2}$ and $M$ along $F_{2}$ and $\Sigma_{h^{\prime}}$ :

$$
Z=W_{2}-n b d\left(F_{2}\right) \cup_{\tilde{\phi}} M-n b d\left(\Sigma_{h^{\prime}}\right) .
$$

Observe that we have taken the symplectic sum along $\Sigma_{h^{\prime}}$, not $F$. Thus $F$ survives as a symplectic genus 2 surface in $Z$.

We choose the base point preserving diffeomorphism $\phi: F_{2} \rightarrow \Sigma_{h^{\prime}}$ to form this sum as follows. Using Lemma 9 in each half of the decomposition of $\Sigma$ into two punctured tori, we conclude that there is a base point preserving diffeomorphism (a composition of six Dehn twists) $\phi_{1}: \Sigma_{h^{\prime}} \rightarrow \Sigma_{h^{\prime}}$ that induces the isomorphism $a_{1}, b_{1}, a_{2}, b_{2}$ to $b_{1}^{-1}, b_{1} a_{1} b_{1}^{-1}, b_{2}^{-1}, b_{2} a_{2} b_{2}^{-1}$ on $\pi_{1}\left(\Sigma_{h^{\prime}}, h^{\prime}\right)$. Composing this with the diffeomorphism $\phi_{2}: F_{2} \rightarrow \Sigma_{h^{\prime}}$ that takes $s_{1}, t_{1}, s_{2}, t_{2}$ to $a_{1}, b_{1}, a_{2}, b_{2}$ yields the desired diffeomorphism $\phi: F_{2} \rightarrow \Sigma_{h^{\prime}}$. Hence $\phi$ induces the isomorphism

$$
\begin{aligned}
\phi: \pi_{1}\left(F_{2}, w\right) & \rightarrow \pi_{1}\left(\Sigma_{h^{\prime}}, h^{\prime}\right), \\
\left(s_{1}, t_{1}, s_{2}, t_{2}\right) & \mapsto\left(b_{1}^{-1}, b_{1} a_{1} b_{1}^{-1}, b_{2}^{-1}, b_{2} a_{2} b_{2}^{-1}\right) .
\end{aligned}
$$

The symplectic manifold $Z$ contains the surface $F$ and six Lagrangian tori $T_{1}^{\prime}, T_{2}^{\prime}, T_{1}, T_{2}, T_{3}, T_{4}$. These seven surfaces are pairwise disjoint. For convenience denote the union of these seven surfaces by $R \subset Z$. Lemma 4 and the discussion following Proposition 6 shows that $\pi_{1}(Z-R)$ is a quotient of $\pi_{1}\left(M-\left(F \cup \cup_{i=1}^{4} T_{i}\right)\right)$. Thus $\pi_{1}(Z-R)$ is generated by $x, y, a_{1}, b_{1}, a_{2}, b_{2}$.

Moreover, the relations of Proposition 6 and Lemma 4 imply that in $\pi_{1}(Z-R)$,

$$
\left[\phi\left(s_{1}\right), \phi\left(s_{2}\right)\right]=\left[\phi\left(t_{1}\right), \phi\left(s_{2}\right)\right]=\left[\phi\left(s_{1}\right), \phi\left(t_{1}\right)\right]=\left[\phi\left(s_{2}\right), \phi\left(t_{2}\right)\right]=1 .
$$

Rewriting this in terms of the $a_{i}, b_{i}$ using Equation (3) one obtains

$$
1=\left[b_{1}^{-1}, b_{2}^{-1}\right], 1=\left[b_{1} a_{1} b_{1}^{-1}, b_{2}^{-1}\right], 1=\left[b_{1}^{-1}, b_{1} a_{1} b_{1}^{-1}\right], 1=\left[b_{2}^{-1}, b_{2} a_{2} b_{2}^{-1}\right] .
$$

Notice that $\left[b_{1}^{-1}, b_{1} a_{1} b_{1}^{-1}\right]=\left[a_{1}, b_{1}\right]$ and $\left[b_{2}^{-1}, b_{2} a_{2} b_{1}^{-1}\right]=\left[a_{2}, b_{2}\right]$. Moreover, $[r, s]=1$ implies $\left[r^{-1}, s^{-1}\right]=1$ and $\left[r, s^{-1}\right]=1$. Hence this set of 
relations simplifies to

$$
1=\left[b_{1}, b_{2}\right], 1=\left[a_{1}, b_{2}\right], 1=\left[b_{1}, a_{1}\right], 1=\left[b_{2}, a_{2}\right] .
$$

Since $\pi_{1}(Z-R)$ is a quotient of $\pi_{1}\left(M-n b d\left(F \cup_{i=1}^{4} T_{i}\right)\right)$, the relations coming from $M-n b d\left(F \cup_{i=1}^{4} T_{i}\right)$ also hold in $\pi_{1}(Z-R)$, hence

$$
\left[x, a_{1}\right]=\left[y, a_{1}\right]=\left[y, b_{1} a_{1} b_{1}^{-1}\right]=\left[x, a_{2}\right]=\left[y, a_{2}\right]=\left[y, b_{2} a_{2} b_{2}^{-1}\right]=1 .
$$

These simplify, using Equation (4), to

$$
1=\left[x, a_{1}\right], 1=\left[y, a_{1}\right], 1=\left[x, a_{2}\right], 1=\left[y, a_{2}\right] .
$$

The following is the result we have been aiming towards. Its usefulness will be illustrated in most of the subsequent constructions in this article.

Theorem 11. Let $Z$ denote the symplectic sum of $W_{2}$ and $M$ along the surfaces $F_{2}$ and $\Sigma_{h^{\prime}}$ using the diffeomorphism $\phi: F_{2} \rightarrow \Sigma_{h^{\prime}}$ inducing the isomorphism of Equation (3) on fundamental groups. Let $R$ denote the union the seven surfaces in $Z$ : the symplectic surface $F$ and the six Lagrangian tori $T_{1}^{\prime}, T_{2}^{\prime}, T_{1}, T_{2}, T_{3}, T_{4}$.

Then the fundamental group of $Z-R$ is generated by the six loops $x, y, a_{1}, b_{1}, a_{2}, b_{2}$. The relations

$$
1=\left[b_{1}, b_{2}\right], 1=\left[a_{1}, b_{2}\right], 1=\left[b_{1}, a_{1}\right], 1=\left[b_{2}, a_{2}\right]
$$

and

$$
1=\left[x, a_{1}\right], 1=\left[y, a_{1}\right], 1=\left[x, a_{2}\right], 1=\left[y, a_{2}\right]
$$

hold in $\pi_{1}(Z-R)$.

The meridian of the surface $F$ is given by $\mu_{F}=[x, y]$. For the six Lagrangian tori, the meridians and Lagrangian push offs (with appropriate paths to the base point) in $\pi_{1}(Z-R)$ are given by:

- $T_{1}^{\prime}: \mu_{1}^{\prime}=\left[a_{2}^{-1}, a_{1}^{-1}\right], m_{1}^{\prime}=b_{1}^{-1}, \ell_{1}^{\prime}=b_{2}^{-1}$.

- $T_{2}^{\prime}: \mu_{2}^{\prime}=\left[b_{1}, a_{2}\right], m_{2}^{\prime}=b_{1} a_{1} b_{1}^{-1}=a_{1}, \ell_{2}^{\prime}=b_{2}^{-1}$.

- $T_{1}: \mu_{1}=\left[b_{1}^{-1}, y^{-1}\right], m_{1}=x, \ell_{1}=a_{1}$.

- $T_{2}: \mu_{2}=\left[x^{-1}, b_{1}\right], m_{2}=y, \ell_{2}=a_{1}$.

- $T_{3}: \mu_{3}=\left[b_{2}^{-1}, y^{-1}\right], m_{3}=x, \ell_{3}=a_{2}$.

- $T_{4}: \mu_{4}=\left[x^{-1}, b_{2}\right], m_{4}=y, \ell_{4}=a_{2}$.

Proof. Proposition 7 implies that the meridian of $F$ in $\pi_{1}(Z-R)$ is given by

$$
\mu_{F}=[x, y] .
$$

Propositions 6 and 7 give the meridians and Lagrangian push offs of the six tori in terms of these generators.

Proposition 6 shows that $\mu_{1}^{\prime}=\left[b_{2} a_{2}^{-1} b_{2}^{-1}, b_{1} a_{1}^{-1} b_{1}^{-1}\right]$. Equation (4) can be used to simplify this: $\left[b_{2} a_{2}^{-1} b_{2}^{-1}, b_{1} a_{1}^{-1} b_{1}^{-1}\right]=\left[a_{2}^{-1}, a_{1}^{-1}\right]$, and hence $\mu_{1}^{\prime}$ is equal to $\left[a_{2}^{-1}, a_{1}^{-1}\right]$. Similarly $\left[b_{1}, b_{2} a_{2} b_{2}^{-1}\right]=\left[b_{1}, a_{2}\right]$, showing that $\mu_{2}^{\prime}=\left[b_{1}, a_{2}\right]$. Next, $\left(b_{2} a_{2} b_{2}^{-1}\right) b_{2}^{-1}\left(b_{2} a_{2} b_{2}^{-1}\right)^{-1}=b_{2}^{-1}$, showing that 
$\ell_{2}^{\prime}=b_{2}^{-1}$. Continuing, $b_{1} a_{1} b_{1}^{-1}=a_{1}$ and so $\ell_{2}=a_{1}$ and $b_{2} a_{2} b_{2}^{-1}=a_{2}$, so that $\ell_{4}=a_{2}$.

The rest of the argument was described before the statement of the Theorem.

q.e.d.

We determine the basic homological properties of $Z$.

Proposition 12. The first homology $H_{1}(Z) \cong \mathbb{Z}^{6}$, generated by the loops $x, y, a_{1}, b_{1}, a_{2}, b_{2}$.

The second homology $H_{2}(Z) \cong \mathbb{Z}^{16}$. There exist six disjoint tori $R_{1}^{\prime}, R_{2}^{\prime}, R_{1}, R_{2}, R_{3}$, and $R_{4}$, and a genus 2 surface $H_{3}$ with trivial normal bundles in $Z$ which are geometrically dual to the six Lagrangian tori $T_{1}^{\prime}, T_{2}^{\prime}, T_{1}, T_{2}, T_{3}$, and $T_{4}$ and the surface $F$, in the sense that $T_{i}^{\prime}$ intersects $R_{i}^{\prime}$ transversally in one point and similarly for $T_{i}, R_{i}$ and $F, H_{3}$, and all other intersections are pairwise empty.

There are two disjoint tori $H_{1}, H_{2}$ with square -1 which intersect $F$ transversally once and are pairwise disjoint from the $T_{i}^{\prime}, R_{i}^{\prime}, T_{i}, R_{i}$, and $H_{3}$. The sixteen surfaces $T_{i}^{\prime}, R_{i}^{\prime}, T_{i}, R_{i}, F, H_{1}, H_{2}$ and $H_{3}$ generate $H_{2}(Z)$. Hence the intersection form of $Z$ is an orthogonal sum of six hyperbolic summands generated by the pairs $T_{i}, R_{i}$ (and $T_{i}^{\prime}, R_{i}^{\prime}$ ) and one 4-dimensional summand spanned by $H_{1}, H_{2}, H_{3}$ and $F$ with matrix

$$
\left(\begin{array}{cccc}
-1 & 0 & 0 & 1 \\
0 & -1 & 0 & 1 \\
0 & 0 & 0 & 1 \\
1 & 1 & 1 & 0
\end{array}\right)
$$

The signature $\sigma(Z)$ equals -2 . The Euler characteristic e $(Z)$ equals 6 .

Moreover, the symplectic manifold $Q$ obtained by performing any six Luttinger surgeries on the $T_{i}^{\prime}$ and $T_{i}$ satisfies $e(Q)=6$ and $\sigma(Q)=-2$, and contains a symplectic genus 2 surface $F$ with trivial normal bundle and six Lagrangian tori.

Proof. Since $Z$ is the symplectic sum of $W_{2}=T^{4} \# 2 \overline{\mathbb{C P}}^{2}$ and $M=$ $T^{2} \times \Sigma$ along genus 2 surfaces,

$$
e(Z)=e\left(T^{4} \# 2 \overline{\mathbb{C P}}^{2}\right)+e\left(T^{2} \times \Sigma\right)+4=6
$$

and Novikov additivity implies that

$$
\sigma(Z)=\sigma\left(T^{4} \# 2 \overline{\mathbb{C P}}^{2}\right)+\sigma\left(T^{2} \times \Sigma\right)=-2 .
$$

Luttinger surgery does not affect $e$ and $\sigma$, and so $e(Q)=6$ and $\sigma(Q)=-2$. The core torus $T \times\{0\} \subset T^{2} \times D^{2}$ glued in to form a Luttinger surgery is Lagrangian [21]. Since the $T_{i}^{\prime}$ and $T_{i}$ miss $F$, the assertions about $Q$ are verified.

The homomorphism $H_{1}\left(F_{2}\right) \rightarrow H_{1}\left(W_{2}-F_{2}\right)=H_{1}\left(W_{2}\right) \cong \mathbb{Z}^{4}$ induced by any push off is an isomorphism. Also $M-\operatorname{nbd}\left(\Sigma_{h^{\prime}}\right)=H \times \Sigma$, where 
$H$ is a punctured torus. Thus from the Mayer-Vietoris sequence for the decomposition

$$
Z=W_{2}-n b d\left(F_{2}\right) \cup_{\tilde{\phi}} M-n b d\left(\Sigma_{h^{\prime}}\right)
$$

one easily sees that $H_{1}\left(M-n b d\left(\Sigma_{h^{\prime}}\right)\right) \rightarrow H_{1}(Z)$ is an isomorphism. Since $H_{1}\left(M-n b d\left(\Sigma_{h^{\prime}}\right)\right)=H_{1}(H \times \Sigma) \cong \mathbb{Z}^{6}$, and $\pi_{1}(H \times \Sigma)$ is generated by the six loops $x, y, a_{1}, b_{1}, a_{2}, b_{2}, H_{1}(Z) \cong \mathbb{Z}^{6}$ generated by these loops. Since $e(Z)=6$, it follows that $H_{2}(Z) \cong \mathbb{Z}^{16}$.

We find 16 embedded surfaces that generate $H_{2}(Z)$ and calculate the intersection form.

The torus $T_{1}$ has a geometrically dual torus $R_{1}=y \times b_{1}$ in $M=\hat{H} \times \Sigma$ which misses $T_{2}, T_{3}, T_{4}$ and also $F$ and its parallel $\Sigma_{h^{\prime}}$ (since the points $u$ and $h^{\prime}$ are different from $h$ ). Similarly $T_{2}$ has a dual torus $R_{2}=x \times b_{1}$, $T_{3}$ has a dual torus $R_{3}=y \times b_{2}$, and $T_{4}$ has a dual torus $R_{4}=x \times b_{2}$. These all miss $F$ and $\Sigma_{h^{\prime}}$ and so survive in $Z$. It is also easy to push the $R_{i}$ off each other. Notice also that the $T_{i}$ and $R_{i}$ miss $\hat{H} \times\{q\}$ for most points $q$.

Similarly one can find disjoint dual tori $R_{1}^{\prime}$ and $R_{2}^{\prime}$ to the classes $T_{1}^{\prime}$ and $T_{2}^{\prime}$ in $W_{2}-F_{2}$. The tori $T_{i}^{\prime}$ and $R_{i}^{\prime}$ may be assumed to miss the 2 exceptional curves $E_{1}$ and $E_{2}$, and also miss one of the vertical tori $\{r\} \times T^{2}$.

Let $H_{1} \subset Z$ be the torus formed by joining up the exceptional curve $E_{1}$ to $H \times\{q\}$ for the appropriate $q$ in the symplectic sum. Similarly define $\mathrm{H}_{2}$ using the other exceptional curve, and let $H_{3}$ be a genus 2 surface formed by joining $H \times\{r\}$ to one of the vertical tori.

The 12 tori $T_{i}, R_{i}, T_{i}^{\prime}, R_{i}^{\prime}$ can be isotoped by a small isotopy in $Z$ so each meets its dual transversally once and all other intersections are empty. Moreover, they each miss $F, H_{1}, H_{2}$, and $H_{3}$. Notice that each $H_{i}$ intersects $F$ once (geometrically and algebraically), that $F^{2}=0$, $H_{1}^{2}=-1, H_{2}^{2}=-1$, and $H_{3}^{2}=0$. Finally $H_{i}$ is disjoint from $H_{j}$ when $i \neq j$. One can check that the classes $F=H_{0}, H_{1}, H_{2}, H_{3}$ span a primitive subspace of $H_{2}(Z)$ by calculating that the determinant of $H_{i} \cdot H_{j}$ is equal to 1 .

Thus the intersection form of $Z$ is the orthogonal sum of 6 hyperbolic planes spanned by each $T_{i}, R_{i}$ pair, and a 4 dimensional space spanned by $F, H_{1}, H_{2}, H_{3}$ with matrix as asserted in the statement.

Notice moreover that the subspace spanned by $F$ and the $T_{i}$ is a 7 dimensional isotropic subspace, and that $H_{2}$ and $H_{3}$ span a subspace with intersection form $2(-1)$.

q.e.d.

We can now do Luttinger surgery on the six tori to obtain interesting symplectic 4-manifolds containing a symplectic genus 2 surface $F$. The effect of Luttinger surgery on the homology is easy to understand. Let $T$ denote one of the $T_{i}$ or $T_{i}^{\prime}$, and $\mu, m, \ell$ its meridian and two Lagrangian 
push offs. Theorem 11 shows that $m$ is sent to one of the generators $x, y, a_{1}, b_{1}, a_{2}, b_{2}$ in $H_{1}(Z)$, and similarly for $\ell$. The meridian $\mu$ is trivial in $H_{1}(Z)$. Therefore,

1) 1/0 surgery on $T$ along any curve $\gamma$ does not change anything, one removes a neighborhood of $T$ and re-glues it the same way.

2 ) For $k \neq 0,1 / k$ surgery on $T$ along the curve $\gamma=m^{p} \ell^{q}$ kills $k(p[m]+q[\ell])$ in $H_{1}(Z)$. The surgery decreases the rank of $H_{1}(Z ; \mathbb{Q})$ by one. Since the Euler characteristic is unchanged, the rank of $H_{2}(Z ; \mathbb{Q})$ decreases by two. If $k= \pm 1$, then the rank of $H_{2}(Z ; \mathbb{Z})$ decreases by two.

Notice that $\pm 1 / 1$ Luttinger surgery on $T$ only changes the manifold near $T$. Letting $\tilde{T}$ denote the core $T \times\{0\}$ of $T \times D^{2}$ in the surgered manifold, we see that $\tilde{T}$ is nullhomologous, since the other surfaces $T_{i}$, $R_{i}, H_{i}$ and $F$ are disjoint from $T$ and its dual and hence are not affected. In other words, the effect on second homology of \pm 1 Luttinger surgery on $T_{i}$ or $T_{i}^{\prime}$ is to kill the hyperbolic summand spanned by $T_{i}$ and its dual.

Computing fundamental groups is harder, but the following refinement of the main result of $[6]$ indicates why we chose the gluing map $\phi$ as we did.

Theorem 13. There exists a minimal symplectic 4-manifold $X$ homeomorphic but not diffeomorphic to $\mathbb{C P}^{2} \# 3 \overline{\mathbb{C P}}^{2}$ containing a symplectic surface $F$ of genus 2 with simply connected complement and trivial normal bundle.

Moreover, $X$ contains a nullhomologous Lagrangian torus $T$ disjoint from $F$, and a curve $\lambda$ on the boundary of the tubular neighborhood of $T$ such that $\lambda=0$ in $H_{1}(X-T)$ and $\lambda$ is isotopic in $n b d(T)$ to an embedded essential curve on $T$.

Proof. We perform six Luttinger surgeries on the six Lagrangian tori in $Z-F$, referring to Theorem 11 . First $1 / 1$ surgery on $T_{1}^{\prime}$ along $m_{1}^{\prime}$ imposes the relation $b_{1}=\left[a_{2}^{-1}, a_{1}^{-1}\right]$. Then $-1 / 1$ surgery on $T_{1}$ along $m_{1}$ imposes the relation $x=\left[b_{1}^{-1}, y^{-1}\right]=\left[\left[a_{1}^{-1}, a_{2}^{-1}\right], y^{-1}\right]=1$. The last equality comes from Equation (5).

Next perform $-1 / 1$ Luttinger surgery on $T_{2}$ along $\ell_{2}$. This imposes $a_{1}=\left[x^{-1}, b_{1}\right]=1$. Returning to our first surgery we see that $b_{1}=\left[a_{2}^{-1}, a_{1}^{-1}\right]=1$. Continuing, $1 / 1$ surgery on $T_{2}^{\prime}$ along $\ell_{2}^{\prime}$ sets $b_{2}=\left[b_{1}, a_{2}\right]=1$.

Next perform $-1 / 1$ surgery on $T_{3}$ along $\ell_{3}$ to set $a_{2}=1$. We finish with $-1 / 1$ surgery on $T_{4}$ along $m_{4}$ which sets $y=1$.

This kills all the generators, so the resulting symplectic 4-manifold $X$ satisfies $\pi_{1}(X-F)=1$. 
It was explained in the paragraphs preceding Theorem 13 that the six Luttinger fillings kill the first homology, and $H_{2}(X)=\mathbb{Z}^{4}$, and that any of the six core tori in $X$ are nullhomologous. Hence we let $T$ denote the core of the neighborhood glued in the last Luttinger surgery.

Since $T \times D^{2}$ is glued along $T \times \partial D^{2}$ so that $\partial D^{2}$ is identified with the curve $\mu_{4} m_{4}^{-1}$, we can choose an essential embedded curve $\lambda$ on $T$ so that $\lambda \times\{s\}$ is sent to $\mu_{4}=\left[x^{-1}, b_{2}\right]=1$ in $\pi_{1}(X-T)$, and hence $\mu_{4}=0$ in $H_{1}(X-T)$. (Note that since each Luttinger surgery kills one generator of first homology, $\pi_{1}(X-T)=H_{1}(X-T)=\mathbb{Z} y$.)

The minimality of $X$ follows just as in the proofs of Theorems 8 and 10 , using Usher's theorem. Note that $e(X)=e(Z)=6$ and $\sigma(X)=$ $\sigma(Z)=-2$, so that by Freedman's theorem $[\mathbf{1 4}] X$ is homeomorphic to $\mathbb{C P}^{2} \# 3 \overline{\mathbb{C P}}^{2}$. As in the proof of Theorem 8 , Taubes's results imply that $V$ is not diffeomorphic to $\mathbb{C P}^{2} \# 3 \overline{\mathbb{C P}}^{2}$. This completes the proof. q.e.d.

Referring to Proposition 12, we see that the four classes generating $H_{2}(X)$ and diagonalizing the intersection form are $\mathrm{F}+\mathrm{H}_{1}, \mathrm{H}_{1}, \mathrm{H}_{2}-\mathrm{H}_{3}$, $H_{3}-H_{1}-F$. These are represented by smoothly embedded surfaces of genus $3,1,3$, and 5 respectively. Notice that $H_{1}$ has minimal genus, since $X$ cannot contain a smoothly embedded -1 sphere. Similarly $\mathrm{H}_{2}$ has minimal genus.

Remark. The last Luttinger surgery in the proof of Theorem 13, $-1 / 1$ surgery on $T_{4}$, kills the last loop $m_{4}=y$. But one can leave the setting of symplectic manifolds, and define a family of smooth manifolds as follows. Denote by $X_{0}$ the symplectic manifold constructed in the proof of Theorem 13 in the penultimate step. Thus $X_{0}$ is a symplectic manifold with $\pi_{1}\left(X_{0}\right)=\mathbb{Z}$, generated by $y$, and $b^{+}\left(X_{0}\right)=2, b^{-}\left(X_{0}\right)=4$. The torus $T_{4}$ in $X_{0}$ is Lagrangian. The boundary of the tubular neighborhood of $T_{4}$ is a 3 -torus whose fundamental group is generated by the loops $\mu_{4}, m_{4}$ and $\ell_{4}$. In $\pi_{1}\left(X_{0}-T_{4}\right), \mu_{4}=1, m_{4}=y$, and $\ell_{4}=1$.

Let $Y$ denote the manifold obtained from $X_{0}-n b d\left(T_{4}\right)$ by gluing $T^{2} \times D^{2}$ in such a way that $\alpha=S^{1} \times\{1\} \times\{1\}$ is sent to $\ell_{4}, \beta=$ $\{1\} \times S^{1} \times\{1\}$ is sent to $\mu_{4}$, and $\mu_{Y}=\{(1,1)\} \times \partial D^{2}$ is sent to $m_{4}^{-1}$. Then $Y$ is simply connected, since $m_{4}=y$ is killed, but $Y$ need not be symplectic. Let $\tilde{T} \subset Y$ denote the resulting core torus.

With these coordinates $\alpha, \beta, \mu_{Y}$, one sees that

1) $X_{0}$ is obtained by $0 / 1$ surgery on $\tilde{T}$ along $\beta$.

2) $\beta$ is nullhomologous (indeed nullhomotopic) in $Y-\tilde{T}=X_{0}-T_{4}$, since $\beta=\mu_{4}$.

3) The manifold $Y_{n}$ obtained from $1 / n$ surgery on $\tilde{T}$ along $\beta$ is simply connected, and hence homeomorphic to $\mathbb{C P}^{2} \# 3 \overline{\mathbb{C P}}^{2}$. (This manifold can be described as $-n / 1$ surgery on $T_{4}$ along $m_{4}$ in $X_{0}$, and so $\mu_{4}^{n} m_{4}^{-1}=y^{-1}$ is killed. Thus $X=Y_{1}$.) 
4) The torus $\tilde{T}$ is nullhomologous in $Y$. The reason is the same as in the construction of $X$ : the last torus surgery kills one generator of $H_{1}$, hence two generators of $H_{2}$, the class of $T_{1}$ and its dual $R_{1}$.

5) $X_{0}$ is symplectic, minimal, and has $b^{+}=2$, hence has non-trivial Seiberg-Witten invariant by [37].

The Morgan-Mrowka-Szabo formula [23] (as formulated in [35, Theorem 3.4]) can be used to prove that the family $\left\{Y_{n}\right\}$ contains infinitely many diffeomorphism types, detected by Seiberg-Witten invariants (see [10, Theorem 5.3]). This proves the following.

Corollary 14. Among the manifolds $Y_{n}, n \in \mathbb{Z}$ there exists an infinite family of smooth manifolds homeomorphic to $\mathbb{C P}^{2} \# 3 \overline{\mathbb{C P}}^{2}$ which are pairwise non-diffeomorphic (detected by Seiberg-Witten invariants). Each $Y_{n}$ contains a smoothly embedded genus 2 surface $F$ with trivial normal bundle and simply connected complement.

q.e.d.

As remarked in [33], Seiberg-Witten invariants cannot be used to distinguish more than two minimal symplectic manifolds homeomorphic to $\mathbb{C P}^{2} \# n \overline{\mathbb{C P}}^{2}$ for $n<9$. Thus at most two of the $Y_{n}$ are minimal symplectic manifolds.

4.4. The manifold $X$ is very useful. We illustrate this with a number of constructions next. A quick corollary is the following (cf. [2]).

Corollary 15. The symplectic sum of $X$ with $W_{2}$ along $F$ in $X$ and $F_{2}$ in $W_{2}$ is a minimal symplectic manifold homeomorphic but not diffeomorphic to $3 \mathbb{C P}^{2} \# 7 \overline{\mathbb{C P}}^{2}$.

Proof. The map $\pi_{1}\left(F_{2}\right) \rightarrow \pi_{1}\left(W_{2}\right)$ induced by inclusion is onto, and hence the symplectic sum $S$ of $W_{2}$ and $X$ is simply connected by Lemma 5 . Since $e(S)=e(X)+e\left(W_{2}\right)+4=12$ and $\sigma(S)=$ $\sigma(X)+\sigma\left(W_{2}\right)=-4$, Freedman's theorem [14] implies that $S$ is homeomorphic to $3 \mathbb{C P}^{2} \# 7 \overline{\mathbb{C P}}^{2}$.

The Seiberg-Witten invariants of a connected sum of manifolds with $b^{+}>1$ vanishes $([\mathbf{3 1}])$. On the other hand the Seiberg-Witten invariants for a symplectic manifold with $b^{+}>1$ are non-trivial $([\mathbf{3 7}])$. Hence $S$ cannot be diffeomorphic to $3 \mathbb{C P}^{2} \# 7 \overline{\mathbb{C P}}^{2}$. $\quad$ q.e.d.

Similarly one obtains the following (cf. [34]).

Corollary 16. The fiber sum of $X$ with $W_{1}$ along $F$ and $F_{1}$ is a minimal symplectic manifold homeomorphic but not diffeomorphic to $3 \mathbb{C P}^{2} \# 9 \overline{\mathbb{C P}}^{2}$.

q.e.d.

And also

Corollary 17. The fiber sum of two copies of $X$ along $F$ is a minimal symplectic manifold homeomorphic but not diffeomorphic to $5 \mathbb{C P}^{2} \# 9 \overline{\mathbb{C P}}^{2}$.

q.e.d. 
In Corollaries 15, 16, and 17 the manifolds constructed contain nullhomologous Lagrangian tori with an appropriate curve to surger, and so the argument given above for $X$ applies to find infinitely many smooth homeomorphic but not diffeomorphic (and not necessarily symplectic) examples in each case.

4.5. Any simply connected symplectic 4 -manifold $(M, \omega)$ containing a symplectic torus of square zero either has $e \geq 12$ or $b^{+} \geq 3$. Indeed, suppose that $b^{+}(M)=1$ and $e<12$. Let $t \in H^{2}(M ; \mathbb{R})$ denote the Poincaré dual to $T$ and $k$ the canonical class. Since $T$ is symplectic, $\omega \cdot t>0$ and $k \cdot t=2 g(T)-2+t \cdot t=0$. Since $k^{2}=2 e+3 \sigma$, it follows from $e<12$ that $k^{2}>0$. Clearly $\omega^{2}>0$. These facts easily imply that the intersection form has a 2-dimensional positive definite subspace, contradicting $b^{+}(M)=1$.

Thus the small simply connected symplectic manifold $X$ with $b^{+}=1$ constructed above cannot contain a square zero torus with simply connected complement.

The elliptic surface $E(1)$, which has $e=12$ and $\sigma=-8$, does contain a symplectic square zero torus with simply connected complement, namely the fiber in any elliptic fibration with cusp fibers. The following theorem establishes the existence of a fake $3 \mathbb{C P}^{2} \# 5 \overline{\mathbb{C P}}^{2}$ which contains a square zero symplectic torus with simply connected complement. In the statement $X$ denotes the manifold of Theorem 13 and $M$ the manifold of Proposition 7. Recall that $X$ contains a square zero genus 2 surface $F$ with simply connected complement. Also, $M$ contains a square zero genus two surface, also denoted $F$, and four homologically essential Lagrangian tori.

Theorem 18. One can do Luttinger surgeries on the symplectic sum of $X$ with $M$ to obtain a minimal symplectic manifold $B$ homeomorphic but not diffeomorphic to $3 \mathbb{C P}^{2} \# 5 \overline{\mathbb{C P}}^{2}$. This manifold contains a pair of tori with trivial normal bundles $T_{3}, T_{4}$, with $B-\left(T_{3} \cup T_{4}\right)$ simply connected. The tori $T_{3}$ and $T_{4}$ can be assumed to be either Lagrangian or symplectic.

Proof. Call the four Lagrangian tori in the sum

$$
A=X-n b d(F) \cup M-n b d(F)
$$

$T_{1}, T_{2}, T_{3}, T_{4}$ (these lie in the $M$ side). Since $X-n b d(F)$ is simply connected, and the boundary of $M-n b d(F)$ carries the loops $a_{1}, b_{1}, a_{2}, b_{2}$, $\pi_{1}(A)$ is generated by the loops $x$ and $y$ in $M$. Proposition 7 shows that the meridians of $T_{1}$ and $T_{2}$ are trivial. Then $-1 / 1$ Luttinger surgery along $m_{1}$ in $T_{1}$ and $m_{2}$ in $T_{2}$ (and no surgery, i.e. 1/0, surgery on $T_{3}$ and $T_{4}$ ) yields a simply connected, symplectic manifold $B$ with $e=10$ and $\sigma=-2$. 
Notice that $\pi_{1}\left(B-\left(T_{3} \cup T_{4}\right)\right)=1$. The Lagrangian torus $T_{3}$ is homologically essential in $B$, since it intersects the $y \times b_{2}$ torus transversally once. Thus one can perturb the symplectic structure on $B$ so that $T_{3}$ becomes symplectic. Similarly for $T_{4}$.

The manifold $X$ is minimal by Theorem 13 . Since it is simply connected, it is not an $S^{2}$ bundle over $F$. The symplectic manifold $M^{\prime}$ obtained from the Luttinger surgeries on $T_{1}$ and $T_{2}$ in $M$ is minimal by the same argument as in the proof of Theorem 10. Since $B$ is the fiber sum of $X$ and $M^{\prime}$, Usher's theorem implies that $B$ is minimal. q.e.d.

The nullhomologous tori in $B$ coming from $T_{1}$ and $T_{2}$ can be used to produce infinitely many non-diffeomorphic smooth manifolds homeomorphic to $3 \mathbb{C P}^{2} \# 5 \overline{\mathbb{C P}}^{2}$, using the argument of Corollary 14. Moreover, the tori $T_{3}$ and $T_{4}$ with simply connected complement can be used to perform knot surgery in the sense of [11].

More simply connected examples can be obtained by taking the symplectic sums with $V$ or $B$. For example, the symplectic sum of $B$ with $W_{1}$ along a parallel copy of $F$ and $F_{1}$ is simply connected by Lemma 4. It is minimal, symplectic, and homeomorphic but not diffeomorphic to $5 \mathbb{C P}^{2} \# 11 \overline{\mathbb{C P}}^{2}$. Similarly the symplectic sum of $B$ with $W_{2}$ gives a minimal symplectic exotic $5 \mathbb{C P}^{2} \# 9 \overline{\mathbb{C P}}^{2}$, the symplectic sum of $V$ with $W_{1}$ gives a minimal symplectic exotic $3 \mathbb{C P}^{2} \# 11 \overline{\mathbb{C P}}^{2}$, and the symplectic sum of $V$ with $W_{2}$ gives a minimal symplectic exotic $3 \mathbb{C P}^{2} \# 9 \overline{\mathbb{C P}}^{2}$ (cf. $[\mathbf{2 6}])$.

This process can be iterated. The following corollary gives one such example.

Corollary 19. Given non-negative integers $m$ and $n$, let $C_{m, n}$ denote the symplectic sum of $X$ with $m$ copies of $W_{1}$ and $n$ copies of $W_{2}$ along parallel copies of $F$. Then $C_{m, n}$ is a simply connected minimal symplectic manifold homeomorphic but not diffeomorphic to the manifold $(1+2 m+2 n) \mathbb{C P}^{2} \#(3+6 m+4 n) \overline{\mathbb{C P}}^{2}$.

Proof. Lemma 4 and induction proves that $C_{m, n}$ is simply connected. We have

$$
e\left(C_{m . n}\right)=e(X)+m e\left(W_{1}\right)+n e\left(W_{2}\right)+4(m+n)=6+8 m+6 n
$$

and

$$
\sigma\left(C_{m, n}\right)=\sigma(X)+m \sigma\left(W_{1}\right)+n \sigma\left(W_{2}\right)=-2(1+2 m+n) .
$$

The intersection form of $C_{m, n}$ is odd for any $n$. To see this, consider the class represented by $\hat{H} \times\{s\}$ in $M=\hat{H} \times \Sigma$. We form $C_{m, n}$ by taking the symplectic sum of $M$ with $m$ copies of $W_{1}$ and $n+1$ copies of $W_{2}$ along $m+n+1$ parallel copies of $F$, say $\left\{p_{i}\right\} \times \Sigma, i=1, \cdots m+n+1$. We can arrange that the first symplectic sum (of $M$ with $W_{2}$ ) is a 
relative symplectic sum $([\mathbf{1 5}])$ which lines up the punctured $\hat{H}$ with one of the exceptional spheres in $W_{2}$. This produces a torus with square -1 in $X$. We can then take relative symplectic sums so that this torus lines up with vertical (square zero) tori in $W_{1}=T^{2} \times S^{2} \# 4 \overline{\mathbb{C P}}^{2}$ and $W_{2}=T^{4} \# 2 \overline{\mathbb{C P}}^{2}$ (rather than the exceptional curves), so that $C_{m, n}$ contains an embedded genus $m+n+1$ surface of square -1 . Hence the intersection form of $C_{m, n}$ is odd.

Using Freedman's theorem we see that $C_{m, n}$ is homeomorphic to the manifold $(1+2 m+2 n) \mathbb{C P}^{2} \#(3+6 m+4 n) \overline{\mathbb{C P}}^{2}$. Minimality is proved as before using Usher's theorem. Taubes's results then imply that $C_{m, n}$ is not diffeomorphic to $(1+2 m+2 n) \mathbb{C P}^{2} \#(3+6 m+4 n) \overline{\mathbb{C P}}^{2}$. q.e.d.

The following theorem is useful to construct manifolds with different smooth structures.

Theorem 20. There exists a minimal symplectic manifold $B_{1}$ containing a pair of Lagrangian tori $T_{3}$ and $T_{4}$ and a square zero symplectic genus 2 surface $F$ so that $T_{3}, T_{4}$ and $F$ are pairwise disjoint and

1) $\pi_{1}\left(B_{1}-\left(F \cup T_{3} \cup T_{4}\right)\right)=\mathbb{Z}^{2}$, generated by $t_{1}$ and $t_{2}$.

2) The inclusion $B_{1}-\left(F \cup T_{3} \cup T_{4}\right) \subset B_{1}$ induces an isomorphism on fundamental groups. In particular the meridians $\mu_{F}, \mu_{3}, \mu_{4}$ all vanish in $\pi_{1}\left(B_{1}-\left(F \cup T_{3} \cup T_{4}\right)\right)$.

3) The Lagrangian push offs $m_{3}, \ell_{3}$ of $\pi_{1}\left(T_{3}\right)$ are sent to 1 and $t_{2}$ respectively in the fundamental group of $B_{1}-\left(F \cup T_{3} \cup T_{4}\right)$.

4) The Lagrangian push offs $m_{4}, \ell_{4}$ of $\pi_{1}\left(T_{4}\right)$ are sent to $t_{1}$ and $t_{2}$ respectively in the fundamental group of $B_{1}-\left(F \cup T_{3} \cup T_{4}\right)$.

5) The push off $F \subset B_{1}-\left(F \cup T_{3} \cup T_{4}\right)$ induces a map on fundamental groups with image the subgroup generated by $t_{2}$.

The symplectic structure may be perturbed so that one or both of the tori $T_{3}, T_{4}$ are symplectic.

Proof. Construct $B_{1}$ by starting with the manifold $Z$ of Theorem 11, and doing Luttinger surgeries along four of the Lagrangian tori, just as was done in the proof of Theorem 13. Explicitly, we do 1/1 Luttinger surgery on $T_{1}^{\prime}$ along $m_{1}^{\prime},-1 / 1$ surgery on $T_{1}$ along $m_{1}$, then $-1 / 1$ surgery on $T_{2}$ along $\ell_{2}$, and finally $1 / 1$ surgery on $T_{2}^{\prime}$ along $\ell_{2}^{\prime}$.

The resulting manifold $B_{1}$ has two remaining Lagrangian tori, $T_{3}$ and $T_{4}$, and the same calculation as in the proof of Theorem 13 shows that $\pi_{1}\left(B_{1}-\left(F \cup T_{3} \cup T_{4}\right)\right)$ is generated by $a_{2}$ and $y$ and $\left[a_{2}, y\right]=1$. Moreover, the meridian $\mu_{F}=1$ and also the other two meridians $\mu_{3}=\mu_{4}=1$. The Lagrangian push offs are given by

$$
m_{3}=1, \ell_{3}=a_{2}, m_{4}=y, \ell_{4}=a_{2}
$$

using Theorem 11. 
Thus $\pi_{1}\left(B_{1}-\left(F \cup T_{3} \cup T_{4}\right)\right)$ is a quotient of $\mathbb{Z} a_{2} \oplus \mathbb{Z} y$. But Proposition 12 shows that $H_{1}\left(B_{1}-\left(F \cup T_{3} \cup T_{4}\right)\right)=\mathbb{Z} \oplus \mathbb{Z}$, and so the group $\pi_{1}\left(B_{1}-\left(F \cup T_{3} \cup T_{4}\right)\right)=\mathbb{Z} a_{2} \oplus \mathbb{Z} y$.

The tori $T_{3}$ and $T_{4}$ have dual tori $R_{3}$ and $R_{4}$ by Proposition 12, and so they are homologically essential and linearly independent in second homology. Hence the symplectic form can be perturbed slightly so that one or both of $T_{3}$ and $T_{4}$ are symplectic $([\mathbf{1 5}])$.

Setting $t_{1}=y$ and $t_{2}=a_{2}$ completes the proof. q.e.d.

The following corollary shows that at the expense of a small stabilization, any symplectic manifold containing a square zero symplectic genus 1 or 2 surface can be used to produce infinite families of manifolds with different Seiberg-Witten invariants. Note that if the surface $G$ has genus 1, then a symplectic sum with $E(1)$ and the knot surgery procedure of Fintushel-Stern [11] gives a similar result, at the cost of 12 to $e$ and -8 to $\sigma$. Thus this result can be viewed as an extension (it includes genus 2) and an improvement (the cost to $e$ is 6 or 10). (See also $[\mathbf{1 2}]$.)

Corollary 21. Suppose $M$ is any symplectic manifold which contains a square zero genus 1 or 2 symplectic surface $G$ such that the inclusion $G \subset M$ induces the trivial map on fundamental groups. Then there exists an infinite family of smooth manifolds $M_{n}$ so that $\pi_{1}\left(M_{n}\right)=$ $\pi_{1}(M), e\left(M_{n}\right)=e(M)+2+4 \operatorname{genus}(G), \sigma\left(M_{n}\right)=\sigma(M)-2$, and the Seiberg-Witten invariants of $M_{n}$ are different from the Seiberg-Witten invariants of $M_{m}$ if $m \neq m$. Moreover $M_{1}$ is symplectic.

Proof. This is an application of the gluing formula for Seiberg-Witten invariants of Mrowka-Morgan-Szabo [23], as explained in [35, Theorem 3.4] (see also [10]) and is similar to the argument given in Corollary 14 above.

Starting with $M$, take the symplectic sum of $M$ with the manifold $B_{1}$ of Theorem 20 along $F$ if $G$ has genus 2, and along $T_{3}$ (after making $T_{3}$ symplectic by perturbing the symplectic structure) if $G$ has genus 1 . Call the result $S$. Then $S$ is a symplectic manifold satisfying $e(S)=$ $e(M)+6+4(\operatorname{genus}(G)-1)$ and $\sigma\left(M_{n}\right)=\sigma(M)-2$.

The Seifert-Van Kampen theorem and Theorem 20 implies that the group $\pi_{1}(S)$ is isomorphic to $\pi_{1}(M) * \mathbb{Z} t_{1}$. The inclusion $S-T_{4} \subset S$ induces an isomorphism on fundamental groups. The Lagrangian push offs of $T_{4}$ are $m_{4}=t_{1}$ and $\ell_{4}=1$, and the meridian is $\mu_{4}=1$.

We follow the argument of Corollary 14. Let $M_{0}$ denote the manifold obtained from $S-n b d\left(T_{4}\right)$ by torus filling by $T^{2} \times D^{2}$ in such a way that $\alpha=S^{1} \times\{(1,1)\}$ is sent to $\ell_{4}, \beta=\{1\} \times S^{1} \times\{1\}$ is sent to $\mu_{4}$, and $\mu=\{(1,1)\} \times \partial D^{2}$ is sent to $m_{4}^{-1}$. Then $\pi_{1}\left(M_{0}\right)=\pi_{1}(M)$ since $t_{1}$ is killed. This is not a Luttinger surgery, so $M_{0}$ need not be symplectic. 
Let $\tilde{T} \subset M_{0}$ denote the resulting core torus. Then $M_{0}-n b d(\tilde{T})=$ $S-n b d\left(T_{4}\right)$. With the coordinates $\alpha, \beta, \mu, S$ is obtained from $M_{0}$ by $0 / 1$ surgery on $\tilde{T}$ along $\beta=\mu_{4}$. Moreover, $\beta$ is nullhomologous in $M_{0}-\tilde{T}$ since $\mu_{4}=0$ in $H_{1}\left(B_{1}-\left(F \cup T_{3} \cup T_{4}\right)\right)$. Note that $S$ is a symplectic manifold. Moreover, using the surfaces described in Proposition 12, since $T_{3}$ has a dual torus $R_{3}$ and either $T_{4}$ also has a dual torus $R_{4}$ (in case $G$ has genus 2) or $F$ has a dual genus 2 surface $H_{3}$ (when $G$ has genus 1), the intersection form of $S$ contains at least 2 hyperbolic pairs, and thus $b^{+}(S)>1$. Hence the Seiberg-Witten invariants of $S$ are non-zero by Taubes's theorem [37].

Since the surgery on $T_{4}$ producing $M_{0}$ from $S$ kills the generator $t_{i}$ of $H_{1}$, it also kills the hyperbolic pair containing $T_{4}$, i.e. $\tilde{T}=0$ in $H_{2}\left(M_{0}\right)$.

Let $M_{n}$ denote the manifold obtained from $1 / n$ surgery on $\tilde{T}$ in $M_{0}$ along $\beta$. Note that $M_{1}$ can also be viewed as $-1 / 1$ Luttinger surgery on $T_{4}$ in $S$ along $m_{4}$. Thus $M_{1}$ is symplectic.

The formula [35, Theorem 3.4] can be used as in [10] to prove that the family $M_{n}$ obtained from $1 / n$ surgery on $\tilde{T}$ along $\beta$ contains infinitely many diffeomorphism types.

q.e.d.

\section{Non-trivial fundamental group}

5.1. Fundamental group $\mathbb{Z}$. We turn now to a useful example of a symplectic 4 -manifold $X_{1}$ with fundamental group $\mathbb{Z}$.

Theorem 22. There exists a minimal symplectic 4-manifold $X_{1}$ with $\pi_{1}\left(X_{1}\right)=\mathbb{Z}, e\left(X_{1}\right)=6$ and $\sigma\left(X_{1}\right)=-2$. Moreover, $X_{1}$ contains a symplectic surface $F$ of genus 2 and square zero and symplectic torus $T$ of square zero disjoint from $F$ so that $\pi_{1}\left(X_{1}-(T \cup F)\right) \cong \mathbb{Z}$. The homomorphisms induced by inclusion, $\pi_{1}\left(X_{1}-(T \cup F)\right) \rightarrow \pi_{1}\left(X_{1}-F\right)$ and $\pi_{1}\left(X_{1}-T\right) \rightarrow \pi_{1}\left(X_{1}\right)$, are isomorphisms.

Furthermore, the homomorphism $\pi_{1}(T) \rightarrow \pi_{1}\left(X_{1}-F\right)$ induced by inclusion takes one generator $t_{1} \in \pi_{1} T$ to the generator of $\pi_{1}\left(X_{1}-F\right)$ and the other $t_{2}$ to the trivial element 1 . The homomorphism induced by inclusion $\pi_{1}(F) \rightarrow \pi_{1}\left(X_{1}\right)$ is trivial.

Proof. We follow the beginning of the proof of Theorem 13, performing Luttinger surgeries on the Lagrangian tori in $Z-F$, where $Z$ is the manifold of Equation (2). We refer to the calculations of Theorem 11.

First $1 / 1$ surgery on $T_{1}^{\prime}$ along $m_{1}^{\prime}$ imposes the relation $b_{1}=\left[a_{2}^{-1}, a_{1}^{-1}\right]$. Then $-1 / 1$ surgery on $T_{1}$ along $m_{1}$ imposes the relation $x=\left[b_{1}^{-1}, y^{-1}\right]=$ $\left[\left[a_{1}^{-1}, a_{2}^{-1}\right], y^{-1}\right]=1$. The last equality comes from Equation (5).

Next perform $-1 / 1$ Luttinger surgery on $T_{2}$ along $\ell_{2}$. This imposes $a_{1}=\left[x^{-1}, b_{1}\right]=1$ and hence also $b_{1}=1$. Then $1 / 1$ surgery on $T_{2}^{\prime}$ along $\ell_{2}^{\prime}$ sets $b_{2}=\left[b_{1}, a_{2}\right]=1$. A $-1 / 1$ surgery on $T_{3}$ along $\ell_{3}$ sets $a_{2}=\left[b_{2}^{-1}, y^{-1}\right]=1$. 
This leaves $T_{4}$ untouched. Call the resulting manifold $X_{1}$. Thus the classes $x, a_{1}, b_{1}, a_{2}$, and $b_{2}$ equal 1 in $\pi_{1}\left(X_{1}-\left(T_{4} \cup F\right)\right)$. Since $H_{1}(Z)=\mathbb{Z}^{6}$ and we performed five Luttinger surgeries, $\pi_{1}\left(X_{1}-\left(T_{4} \cup F\right)\right)$ is generated by $y$ and $H_{1}\left(X_{1}\right)=\mathbb{Z}$, so that $\pi_{1}\left(X_{1}-\left(T_{4} \cup F\right)\right)=\pi_{1}\left(X_{1}-F\right)=$ $\pi_{1}\left(X_{1}\right)=\mathbb{Z} y$.

Then the torus $T_{4}$ carries the classes $y$ and $a_{2}$, and the surface $F$ is generated by the classes $a_{1}, b_{1}, a_{2}, b_{2}$, and so the assertions about fundamental groups follow. Note that $e\left(X_{1}\right)=e(Z)=6$ and $\sigma\left(X_{1}\right)=$ $\sigma(Z)=-2$.

Lastly, the torus $T_{4}$ meets the torus $Q=x \times b_{2}$ in $M$ transversally in one point. Since $Q$ misses all other tori in the construction and $F$, it survives to provide $T_{4}$ with a dual class in $H_{2}\left(X_{1}\right)$. In particular, $\left[T_{4}\right] \neq 0$ in $H_{2}\left(X_{1}\right)$. Hence the symplectic form on $X_{1}$ can be perturbed slightly so that $T_{4}$ (which we rename $T$ ) becomes symplectic $([\mathbf{1 5}])$. We relabel its generators $t_{1}=y$ and $t_{2}=a_{2}$.

Minimality follows just as in the proof of Theorem $10 . \quad$ q.e.d.

The intersection form of $X_{1}$ is equivalent to $2(1) \oplus 4(-1)$. But $X_{1}$ is not diffeomorphic to $\left(S^{1} \times S^{3}\right) \# 2 \mathbb{C P}^{2} \# 4 \overline{\mathbb{C P}}^{2}$, since $X_{1}$ is a minimal symplectic manifold with $b^{+}=2>1$, and hence has non-vanishing Seiberg-Witten invariants [37]. On the other hand the Seiberg-Witten invariants of the connected sum $\left(S^{1} \times S^{3}\right) \# 2 \mathbb{C P}^{2} \# 4 \overline{\mathbb{C P}}^{2}$ must vanish. It is an interesting question whether the manifold $X_{1}$ is homeomorphic to $\left(S^{1} \times S^{3}\right) \# 2 \mathbb{C P}^{2} \# 4 \overline{\mathbb{C P}}^{2}$.

Our interest in the manifold $X_{1}$ is two fold. First, it is the smallest known (to us) symplectic 4-manifold with fundamental group $\mathbb{Z}$, where we measure the size using the Euler characteristic (or equivalently the second Betti number). (Constructions of symplectic manifolds with fundamental group $\mathbb{Z}$ can be found in the literature, e.g. $[\mathbf{2 4}, \mathbf{1 5}, \mathbf{3 2}]$.) The other reason is that it can be used as a smaller replacement for the elliptic surface $E(1)$ typically used to control fundamental groups of symplectic 4-manifolds. We will illustrate this in the following theorem, which also refers to the manifold $B$ constructed in Theorem 18 .

Theorem 23. Let $L$ be a symplectic 4-manifold containing a symplectic torus $T^{\prime}$ with trivial normal bundle such that $\alpha, \beta \in \pi_{1}(L)$ represent the two generators of $\pi_{1}\left(T^{\prime}\right)$. Then

1) The symplectic sum of $X_{1}$ and $L$ along $T$ and $T^{\prime}, X_{1} \#_{T} L$, using the gluing map $\phi: T \rightarrow T^{\prime}$ inducing

$$
t_{1} \mapsto \alpha, t_{2} \mapsto \beta
$$

admits a symplectic structure which agrees with that of $X_{1}$ and $L$ away from $T, T^{\prime}$ and satisfies

$$
e\left(X_{1} \#_{T} L\right)=e(L)+6, \sigma\left(X_{1} \#_{T} L\right)=\sigma(L)-2,
$$


and

$$
\pi_{1}\left(X_{1} \#_{T} L\right)=\pi_{1}(L) / N(\beta)
$$

where $N(\beta)$ denotes the normal subgroup of $\pi_{1}(L)$ generated by $\beta$.

2) The symplectic sum of $B$ and $L$ along $T_{3}$ and $T^{\prime}, B \#_{T} L$ admits a symplectic structure which agrees with that of $B$ and $L$ away from $T_{3}, T^{\prime}$ and satisfies

$$
e\left(B \#_{T} L\right)=e(L)+10, \sigma\left(B \#_{T} L\right)=\sigma(L)-2,
$$

and

$$
\pi_{1}\left(B \#_{T} L\right)=\pi_{1}(L) / N(\alpha, \beta) .
$$

3) Suppose $L$ is a symplectic 4-manifold and $\tilde{F} \subset L$ is a genus 2 symplectic surface with trivial normal bundle. Then the symplectic sum $L \#_{F} X$ of $L$ and $X$ along their genus 2 surfaces is a symplectic manifold satisfying

$$
e\left(L \#_{F} X\right)=e(L)+10, \sigma\left(L \#_{F} X\right)=\sigma(L)-2
$$

and

$$
\pi_{1}\left(L \# \#_{F} X\right)=\pi_{1}(L) / N
$$

where $N$ denotes the normal subgroup of $\pi_{1}(L)$ generated by the image of $\pi_{1}(\tilde{F}) \rightarrow \pi_{1}(L)$.

Proof. The assertions about $e$ and $\sigma$ are straightforward. The fundamental group assertion is proved using the Seifert-Van Kampen theorem.

q.e.d.

Theorem 23 can be restated informally by saying that at a cost of 6 to the Euler characteristic one can symplectically kill one class in the fundamental group of a symplectic manifold, provided that class is carried by a symplectic torus. Similarly at a cost of 10 to $e$ one can kill two classes carried on a symplectic torus. Lastly at a cost of 10 to $e$ one can kill four classes carried by a symplectic genus 2 surface.

5.2. Arbitrary fundamental group. The fundamental group $G$ of a closed, orientable 4-manifold $M$ determines all its Betti numbers except $b_{2}(M)$. Moreover, by the Hopf exact sequence, $b_{2}(M) \geq b_{2}(G)$, and hence

$$
e(M)=2-2 b_{1}(M)+b_{2}(M)=2-2 b_{1}(G)+b_{2}(M) \geq 2-2 b_{1}(G)+b_{2}(G) .
$$

In particular, thinking of $b_{2}(M)$ as a measure of the size of a 4manifold $M$ among manifolds with a given fundamental group $G$, one sees that minimizing the Euler characteristic is the same as minimizing this size, One can get more subtle lower bounds on $e(M)$ than (6) by studying the algebraic topology (e.g. the ring structure) of $K(G, 1)$; see $[17,18]$. 
As explained in Section 4 of [5], the existence of the symplectic manifold $X_{1}$ and its symplectic torus $T$ of Theorem 23 allows us to improve (by 50\%) the main result of [5] to the following theorem.

Theorem 24. Let $G$ be a finitely presented group that has a presentation with $g$ generators and $r$ relations. Then there exists a symplectic 4-manifold $M$ with $\pi_{1} M \cong G$, Euler characteristic e $(M)=10+6(g+r)$, and signature $\sigma(M)=-2-2(g+r)$.

Proof. The proof relies on our construction in [5, Theorem 6] of a symplectic 4-manifold $N$ whose fundamental group contains classes $s, t, \gamma_{1}, \cdots, \gamma_{r+g}$ so that

$$
G \cong \pi_{1}(N) / N\left(s, t, \gamma_{1}, \cdots, \gamma_{r+g}\right)
$$

where $N\left(s, t, \gamma_{1}, \cdots, \gamma_{r+g}\right)$ denotes the normal subgroup generated by the classes $s, t, \gamma_{1}, \cdots, \gamma_{r+g}$.

Moreover, $N$ contains symplectic tori $T_{0}, T_{1}, \cdots, T_{g+r}$ so that the two generators of $\pi_{1}\left(T_{0}\right)$ represent $s$ and $t$, and for $i \geq 1$ the two generators of $\pi_{1}\left(T_{i}\right)$ represent $s$ and $\gamma_{i}$. The manifold $N$ satisfies $e(N)=0$ and $\sigma(N)=0$; in fact $N$ is a product $Y \times S^{1}$ where $Y$ is a 3-manifold that fibers over $S^{1}$.

Let $B$ denote the manifold of Theorem 18. Take the fiber sum of $N$ with $B$ along $T_{0}$, and $g+r$ copies of the manifold $X_{1}$ of Theorem 22 along the tori $T_{i}, i \geq 1$ using an appropriate gluing map as in Theorem 23 , so that $s, t$ and the $\gamma_{i}$ are killed. Then a repeated application of Theorem 23 computes

$$
\pi_{1}(M)=\pi_{1}(N) / N\left(s, t, \gamma_{i}\right)=G, e(M)=10+6(g+r),
$$

and

$$
\sigma(M)=-2-2(g+r) .
$$

q.e.d.

An examination of the proof of Theorem 6 of [5] shows that Theorem 24 can be improved for certain presentations, namely, if $G$ is the quotient of a surface group, $G=\left\langle x_{i}, y_{i} \mid \prod\left[x_{i}, y_{i}\right], w_{1}, \cdots, w_{r}\right\rangle$, one can find $M$ so that $e(M)=10+6\left(2 g^{\prime}+r\right)$ and $\sigma(M)=-2-2\left(2 g^{\prime}+r\right)$, where $g^{\prime}$ is the number of generators which appear in some relation $w_{i}$ with negative exponent.

Indeed, the first step of the construction of [5, Theorem 6] replaces a presentation of $G$ by a new presentation in which every relation is expressed as a word in the generators with only positive exponents. One way to do this is by adding a pair of generators $\tilde{a}, \tilde{b}$ and two relations $a \tilde{a}=1$ and $\tilde{b}=1$ for each generator $a$ which appears in the first presentation with negative exponent in some relation. In the new presentation, any occurrence of $a$ with a negative exponent in a relation is replaced by $\tilde{a}$. This process produces a presentation of $G$ as a quotient of a surface 
group with $r+2 g^{\prime}$ relations in which every generator appears with only positive powers in each relation. The approach used in Theorem 24 is to add a generator $\tilde{a}$ and a relation $a \tilde{a}=1$ for every generator $a$ of the first presentation.

In particular, if $G$ is a quotient of a surface group with $r$ additional relations in which every generator appears only with positive exponent in each additional relation, then there exists a symplectic 4-manifold $M$ with fundamental group $\pi_{1}(M)=G$, and $e(M)=10+6 r, \sigma(M)=-2-$ $2 r$. Moreover, using Usher's theorem [39] one sees that the manifolds constructed are minimal.

Variations on this idea show that if $G$ is the free product of $n$ finite cyclic groups, then $G$ has a presentation

$$
G=\left\langle x_{1}, \cdots, x_{n} \mid x_{1}^{a_{1}}, \cdots, x_{n}^{a_{n}}\right\rangle
$$

with all the $a_{i}>0$, and there exists a symplectic 4-manifold with fundamental group $G$ and $e=10+6 n$.

If one uses $E(1)$ instead of $B$ in the proof of Theorem 24 , the resulting manifold has $e=12+6(g+r)$ and $\sigma=-8-2(g+r)$, and contains a symplectic torus which lies in a cusp neighborhood. Thus the geography results of J. Park [28] can be improved to find a larger region of the $\left(c_{1}^{2}, \chi_{h}\right)$ plane for which to each pair of integers in that region one can find infinitely many non-diffeomorphic, homeomorphic minimal symplectic manifolds with fundamental group $G$.

Another (decidedly minor) improvement concerns groups of the form $G \times \mathbb{Z}$ : for a presentation of $G$ as above there exists a symplectic manifold $M$ with $\pi_{1}(M)=G \times \mathbb{Z}, e(M)=6(g+r+1)$, and $\sigma(M)=-2(g+r+1)$. The reason is that one step in the proof of [5, Theorem 6] consists of taking a symplectic sum with $E(1)$ to kill two generators ( $t$ and $s$ along $T_{0}$ in the notation of the proof of Theorem 24). But to get $G \times \mathbb{Z}$ it suffices to kill $t$, for which the manifold $X_{1}$ can be used instead of $B$, using Theorem 23. This only adds 6 to $e$.

Notice that if $G$ is the fundamental group of a 3-manifold $Y$ that fibers over $S^{1}$, then $Y \times S^{1}$ is a symplectic 4-manifold with fundamental group $G \times \mathbb{Z}$ and Euler characteristic zero.

Suppose $M$ is a symplectic 4-manifold containing a symplectic torus $T$ with trivial normal bundle so that $\pi_{1}(M-T)=1$ or $\mathbb{Z}$ and so that pushing $T$ into $M-T$ induces a surjection $\pi_{1}(T) \rightarrow \pi_{1}(M-T)$. One can prove (using the argument we gave at the beginning of Section 4.5) that if $M$ is simply connected then $e(M) \geq 6$, and if $6 \leq e(M)<12$ then $b^{+}(M)>1$. The manifold $B$ of Theorem 18 is such a simply connected manifold and has $e(B)=10$ and $b^{+}(B)=3$. A similar argument shows that if $\pi_{1}(M) \cong \mathbb{Z}$ then $e(M) \geq 3$, and if $3 \leq e(M)<6$ then $b^{+}(M)>1$. The manifold $X_{1}$ has $e\left(X_{1}\right)=6$ and $b^{+}\left(X_{1}\right)=2$. 
Further improvements in the geography problem for symplectic manifolds will be obtained if such an $M$ is found with $6 \leq e(M)<10$ in the simply connected case and $3 \leq e(M)<6$ in the $\mathbb{Z}$ case. The search for such a manifold is a promising direction for future study.

\subsection{Free groups.}

Theorem 25. For any $n$ there exists a symplectic 4-manifold $D_{n}$ with fundamental group free of rank $n, e\left(D_{n}\right)=10$, and $\sigma\left(D_{n}\right)=-2$.

Proof. This is explained in [5, Theorems 8 and 11], but here, instead of taking a symplectic sum with $E(1)$, one uses the smaller manifold $B$ of Theorem 18. Specifically, let $E$ be a closed surface of genus $n$, with standard generators $x_{1}, y_{1}, \cdots, x_{n}, y_{n}$ of $\pi_{1}(E)$. Let $D: E \rightarrow E$ be the diffeomorphism given by composite of the $n$ Dehn twists along the $x_{i}$ and let $Y$ be the corresponding 3-manifold which fibers over $S^{1}$. Then $Y \times S^{1}$ is a symplectic 4-manifold containing a symplectic torus $T^{\prime}=S \times S^{1}$ where $S$ is a section of $Y \rightarrow S^{1}$.

The standard calculation of $\pi_{1}(Y)$ as an HNN extension shows

$$
\pi_{1}\left(Y \times S^{1}\right)=\left\langle x_{i}, y_{i}, t \mid t x_{i} t^{-1}=x_{i}, t y_{i} t^{-1}=y_{i} x_{i}\right\rangle \oplus \mathbb{Z} s .
$$

By Theorem 23, taking the symplectic sum of $Y \times S^{1}$ with $B$ along $T^{\prime}$ and $T$ has fundamental group obtained by killing $t$ and $s$ in $\pi_{1}\left(Y \times S^{1}\right)$, which also kills the $x_{i}$ (since the relation $y_{i}=y_{i} x_{i}$ implies that $x_{i}=1$ ), leaving the free group generated by the $y_{i}$. Theorem 23 together with $e\left(Y \times S^{1}\right)=0$ and $\sigma\left(Y \times S^{1}\right)=0$ gives the result. $\quad$ q.e.d.

Notice that the smooth 4-manifold obtained by taking connected sums of $n$ copies of $S^{1} \times S^{3}$ has fundamental group free of rank $n$ and $e=2(1-n)$. Kotschick has shown $([\mathbf{2 0}])$ that any symplectic 4-manifold with fundamental group free of rank $n$ has $e \geq \frac{6}{5}(1-n)$. Thus the gap in size (measured say by the rank of the second homology) between the smallest smooth and symplectic manifolds must grow linearly with the rank. The manifold of Theorem 25, with fundamental group free of rank $n$ and $e=10$, is the smallest known symplectic 4-manifold with free fundamental group. With the exception of rank 1 (for which the manifold $X_{1}$ has $e=6$ ) we do not know of any symplectic 4-manifold $M$ with fundamental group free of rank $n$ which satisfies

$$
\frac{6}{5}(1-n) \leq e(M)<10 .
$$

5.4. Fundamental groups associated to surface bundles over the circle. By exactly the same proof as Theorem 25 one establishes (see [5, Theorem 8]).

Theorem 26. If $H: F \rightarrow F$ be an orientation preserving diffeomorphism of a closed orientable surface, fixing a point $z$, and $G$ is the quotient of $\pi_{1}(F, z)$ by the normal subgroup generated by the words 
$x^{-1} H_{*}(x)$, then there exists a symplectic 4-manifold with fundamental group $G$, Euler characteristic $e=10$, and signature $\sigma=-2$.

Moreover, there exists a symplectic 4-manifold with fundamental group $G \times \mathbb{Z}$, Euler characteristic $e=6$, and signature $\sigma=-2 . \quad$ q.e.d.

We can also produce small symplectic 4-manifolds with the same fundamental group as a fibered 3-manifold.

Theorem 27. If $H: F \rightarrow F$ be an orientation preserving diffeomorphism of a closed orientable surface and $Y \rightarrow S^{1}$ the F-bundle over $S^{1}$ with monodromy $H$, then there exists a symplectic 4-manifold $D$ with $\pi_{1}(D)=\pi_{1}(Y), e(D)=6$, and $\sigma(D)=-2$.

Proof. The manifold $Y \times S^{1}$ admits a symplectic structure so that the torus $S \times S^{1}$ is symplectic, where $S \subset Y$ is a section. Theorem 23 shows that taking a symplectic sum of $Y \times S^{1}$ with $X_{1}$ along an appropriate diffeomorphism of tori yields a symplectic manifold $D$ in which the homotopy class of the $S^{1}$ factor is killed, and so $\pi_{1}(D)=\pi_{1}(Y)$. This manifold has $e=6$ and $\sigma=-2$.

q.e.d.

In [18] it is established that for any closed 3-manifold group $G$ and any number $\sigma$, there exists a smooth 4-manifold with fundamental group $G$ and $e=2+|\sigma|$, and that this is the smallest possible Euler characteristic among all 4-manifolds with fundamental group $G$ and signature $\sigma$. In particular, there is a smooth 4-manifold with fundamental group $G$, $\sigma=-2$, and $e=4$. We have found a symplectic 4-manifold whose Euler characteristic is within 2 of the smooth minimum for those $G$ which are the fundamental group of a fibered 3-manifold.

5.5. Free abelian groups. The manifold $X_{1}$ constructed in Theorem 22 is currently the smallest known symplectic 4-manifold with fundamental group infinite cyclic, with $e=6$. Producing small smooth 4manifolds with free abelian fundamental groups poses an interesting challenge $[\mathbf{1 7}, \mathbf{1 8}]$. The number of required relations in a presentation grows quadratically in the number of relations, and so one expects many 2-handles in a handlebody presentation.

Finding symplectic examples is harder. For free abelian groups of even rank a nearly complete answer was found in the collection of symplectic (in fact Kähler) manifolds $\operatorname{Sym}^{2}\left(F_{n}\right)\left(F_{n}\right.$ a surface of genus $n)$. The manifold $\operatorname{Sym}^{2}\left(F_{n}\right)$ has fundamental group $\mathbb{Z}^{2 n}$ and minimizes the Euler characteristic among symplectic manifolds with fundamental group $\mathbb{Z}^{2 n}$ except possibly when $n \equiv 2(\bmod 4)([\mathbf{5}])$.

For odd rank free abelian fundamental group the situation is less clear. We do not know if there exists a symplectic manifold $M$ with fundamental group $\mathbb{Z}$ and $2 \leq e(M)<6$, nor any reason why such a manifold cannot exist. For rank 3, we have the following result. 
Corollary 28. There exists a symplectic 4-manifold $X_{3}$ with fundamental group $\mathbb{Z}^{3}, e=6$ and $\sigma=-2$.

Proof. Start with the manifold $Z$ of Theorem 11. We do a sequence of Luttinger surgeries, as in the proof of Theorems 13 and 22. We first do $1 / 1$ surgery on $T_{1}^{\prime}$ along $m_{1}^{\prime}$ to impose the relation $b_{1}=\left[a_{2}^{-1}, a_{1}^{-1}\right]$. Then $-1 / 1$ surgery on $T_{1}$ along $\ell_{1}$ yields $a_{1}=\left[b_{1}^{-1}, y^{-1}\right]=\left[\left[a_{2}^{-1}, a_{1}^{-1}\right], y^{-1}\right]=1$ using Equation (5). Thus also $b_{1}=1$. Next, $1 / 1$ surgery on $T_{2}^{\prime}$ along $\ell_{2}^{\prime}$ gives $b_{2}=\left[b_{1}, a_{2}\right]=1$. The remaining three generators $x, y, a_{2}$ commute using Equation (5) and the fact that $[x, y]=1$ (we did not remove $F$ ). Call the result $X_{3}$.

Since each surgery decreases the first Betti number by one, and $H_{1}(Z)=\mathbb{Z}^{6}$ it follows that $\pi_{1}\left(X_{3}\right)=H_{1}\left(X_{3}\right)=\mathbb{Z}^{3}$. q.e.d.

The smallest previously known example of a symplectic 4-manifold with fundamental group $\mathbb{Z}^{3}$ has $e=12$. Any such symplectic manifold must have $e \geq 3([\mathbf{5}])$. We know no reason why an example satisfying $3 \leq e \leq 5$ cannot exist.

More generally, the technique of [5, Theorem 20] allows us to improve the construction of symplectic 4-manifolds with odd rank free abelian groups by taking the fiber sum of $\operatorname{Sym}^{2}\left(F_{n}\right)$ with the manifold $X_{1}$ along an appropriate torus, rather than the larger manifold $K$ of $[\mathbf{5}$, Lemma 18]. We refer the interested reader to [5] for details of the proof of the following corollary, which follows by replacing every occurrence of the symbol $K$ by $X_{1}$ in the proof of [5, Lemma 18].

Corollary 29. There exists a symplectic 4-manifold $M$ with $\pi_{1}(M)=$ $\mathbb{Z}^{2 n-1}$ such that $e(M)=9-5 n+2 n^{2}$ and $\sigma(M)=-1-n$. q.e.d.

This is an improvement over the best previously known example, $\left(e(M)=15-5 n+2 n^{2}\right.$ of [5, Theorem 20]), but still far from the lower bound $6-7 n+2 n^{2} \leq \min _{\pi_{1}(M)=\mathbb{Z}^{2 n-1}} e(M)$.

5.6. Abelian groups. Modifying the argument of Corollary 28 gives the following.

Corollary 30. Given any $p, q, r \in \mathbb{Z}$ there exists a symplectic 4manifold $X_{p, q, r}$ with fundamental group $\mathbb{Z} / p \oplus \mathbb{Z} / q \oplus \mathbb{Z} / r$, e $=6$ and $\sigma=-2$.

Proof. Start with the manifold $X_{3}$ of Corollary 28. Thus $\pi_{1}\left(X_{3}\right)=$ $\mathbb{Z}^{3}$, generated by $x, y$, and $a_{2}$, and $b_{1}=a_{1}=b_{2}=1$. The Lagrangian tori $T_{2}, T_{3}$ and $T_{4}$ were not used to construct $X_{3}$. Then $1 / p$ Luttinger surgery on $T_{2}$ along $m_{2}=y$ sets $y^{p}=1$. Similarly $1 / q$ surgery on $T_{3}$ along $m_{3}=x$ and $1 / r$ surgery on $T_{4}$ along $\ell_{4}=a_{2}$ sets $x^{q}=1$ and $a_{2}^{r}=1$.

q.e.d. 
Note that the smallest previously known symplectic 4-manifolds with finite cyclic abelian group are certain complex algebraic surfaces of general type with $e=10[\mathbf{8}]$. For sums of two or three abelian group the smallest previously known examples had $e=12[\mathbf{1 5}, \mathbf{5}]$.

The finitely generated abelian group with $n$ generators

$$
G=\mathbb{Z}^{n-k} \oplus \mathbb{Z} / d_{1} \oplus \mathbb{Z} / d_{2} \oplus \cdots \oplus \mathbb{Z} / d_{k}
$$

(with $d_{i}>1$ ) has a presentation with $n$ generators, and $\left(\begin{array}{l}n \\ 2\end{array}\right)+k$ relations. Thus Theorem 24 implies that there exists a symplectic manifold $N$ with fundamental group $G$ satisfying

$$
e(N)=10+6\left(n+\left(\begin{array}{l}
n \\
2
\end{array}\right)+k\right), \sigma(N)=-2\left(n+\left(\begin{array}{l}
n \\
2
\end{array}\right)+k+1\right) .
$$

The leading term of this expression for $e$ as a function of $n$ is $3 n^{2}$. In other words, if we let

$p(n)=\min \left\{e(N) \mid \pi_{1}(N)\right.$ is abelian and is generated by $n$ elements $\}$

one can say that

$$
\lim _{n \rightarrow \infty} \frac{p(n)}{n^{2}} \leq 3
$$

Theorem 2 of [17] implies that

$$
\frac{1}{2} \leq \lim _{n \rightarrow \infty} \frac{p(n)}{n^{2}}
$$

The manifolds $\operatorname{Sym}^{2}\left(F_{n}\right)$ and those constructed in Corollary 29 show that for free abelian groups with $n$ generators, the leading term for $e$ is $\frac{n^{2}}{2}$. In fact, the following theorem shows this to be true for all finitely generated abelian groups.

Theorem 31. Assume $n$ is even by setting $d_{k}=1$ if necessary. There exists a symplectic manifold $N_{G}$ with fundamental group

$$
G=\mathbb{Z}^{n-k} \oplus \mathbb{Z} / d_{1} \oplus \mathbb{Z} / d_{2} \oplus \cdots \oplus \mathbb{Z} / d_{k}
$$

satisfying

and

$$
e\left(N_{G}\right)=\frac{1}{2} n^{2}+\frac{19}{2} n+36
$$

$$
\sigma\left(N_{G}\right)=-\frac{5}{2} n-8
$$

Proof. Write $G=\mathbb{Z} / d_{1} \oplus \mathbb{Z} / d_{2} \oplus \cdots \oplus \mathbb{Z} / d_{n}$, where we allow $d_{n}$ to be any integer. By allowing $d_{n}=1$ if necessary, we may assume that $n$ is even, say $n=2 g-6$ for some $g \geq 3$.

Let $F_{g}$ denote an compact Riemann surface of genus $g$. The Kähler surface (hence symplectic 4-manifold)

$$
S_{g}=\operatorname{Sym}^{2}\left(F_{g}\right)=F_{g} \times F_{g} / \sim,(x, y) \sim(y, x)
$$


has fundamental group isomorphic to $\mathbb{Z}^{2 g}$.

A pair $x, y$ of embedded curves in $F_{g}$ determine a torus $\tilde{T}(x, y)=x \times y$ in $F_{g} \times F_{g}$. This torus descends to an embedded torus $T(x, y) \subset S_{g}$ when $x \cap y=\phi$. Moreover, in this case $\tilde{T}(x, y)$ is Lagrangian in $F_{g} \times F_{g}$ and its image $T(x, y) \subset S_{g}$ is Lagrangian ([5, Proposition 21], [30]).

Choose embedded curves $a_{1}, b_{1}, a_{2}, \cdots, a_{g}, b_{g} \subset F_{g}$ which represent a standard symplectic basis for $H_{1}\left(F_{g}\right)$. The composite

$$
F_{g} \cong F_{g} \times\{p\} \rightarrow F_{g} \times F_{g} \rightarrow S_{g}
$$

takes $\left\{a_{i}, b_{i}\right\}$ to a basis for $H_{1}\left(S_{g}\right)$. Let $x_{4}, y_{4}, x_{5}, y_{5}, \cdots, x_{g}, y_{g}$ denote $2 g-6$ parallel copies of the curve $a_{1}$ on $F_{g}$. Consider the Lagrangian tori

$$
T\left(a_{1}, b_{2}\right), T\left(b_{1}, b_{3}\right), T\left(a_{2}, a_{3}\right)
$$

and also

$$
T\left(x_{4}, a_{4}\right), T\left(y_{4}, b_{4}\right), T\left(x_{5}, a_{5}\right), T\left(y_{5}, b_{5}\right), \cdots, T\left(x_{g}, a_{g}\right), T\left(y_{g}, b_{g}\right) .
$$

These $2 g-3$ tori are pairwise disjointly embedded in $S_{g}$. They are each homologically essential since each one intersects a dual torus of the same form (e.g. $T\left(x_{i}, a_{i}\right)$ intersects $T\left(b_{1}, b_{i}\right)$ transversally once). Moreover, they are linearly independent since one can check that together with their dual tori they span a hyperbolic subspace of $H_{2}\left(S_{g}\right)$. Thus [15, Lemma1.6] implies that the symplectic form on $S_{g}$ can be perturbed slightly so that these $2 g-3$ tori are symplectic.

Since $\pi_{1}\left(S_{g}\right)$ is abelian, the Hurewicz map to $H_{1}\left(S_{g}\right)$ is an isomorphism, and taking symplectic sums with $X_{1}$ has the same effect on the fundamental group as on the first homology. In particular, we need not worry about base points: killing a class in $H_{1}\left(S_{g}\right)$ also kills the corresponding class in $\pi_{1}\left(S_{g}\right)$.

Now take the symplectic sum of $S_{g}$ with 3 copies of the manifold $B$ and $2 g-6$ copies of the manifold $X_{1}$ as follows. The three copies of $B$ are summed along the three tori of Equation (7). This kills $a_{1}, b_{2}, b_{1}, b_{3}, a_{2}$ and $a_{3}$.

The $2 g-6$ copies of $X_{1}$ are summed along the other tori using appropriate diffeomorphisms. To start, take the sum with $X_{1}$ along $T \subset X_{1}$ the diffeomorphism $T \rightarrow T\left(x_{4}, a_{4}\right)$ which takes the generators $t_{1}, t_{2}$ of $\pi_{1}(T)$ to $a_{4}^{-1}, x_{4} a_{4}^{d_{1}}$. Theorem 23 implies that this kills $x_{4} a_{4}^{d_{1}}$. Recall that $x_{4}$ is a parallel copy of $a_{1}$. Since $a_{1}$ was killed before, this sets $a_{4}^{d_{1}}=1$. Continue by setting $b_{4}^{d_{2}}=1$, etc. Note that $d_{i}$ can be any integer, including 0 or 1.

This produces a symplectic manifold $N_{G}$ with fundamental group $G$. Theorem 23 shows that

$e\left(N_{G}\right)=30+6(2 g-6)+e\left(S_{g}\right)=12 g-6+\left(2 g^{2}-5 g+3\right)=\frac{1}{2} n^{2}+\frac{19}{2} n+36$ 
and

$$
\sigma\left(N_{G}\right)=-2(2 g-3)+\sigma\left(S_{g}\right)=-4 g+6+(1-g)=-\frac{5}{2} n-8 .
$$

q.e.d.

\section{References}

[1] D. Auroux, S. K. Donaldson \& L. Katzarkov, Luttinger surgery along Lagrangian tori and non-isotopy for singular symplectic plane curves, Math. Ann. 326 (2003), no. 1, 185-203, MR 1981618, Zbl 1026.57020.

[2] A. Akhmedov, Exotic smooth structures on $3 \mathbb{C P}^{2} \# 7 \overline{\mathbb{C P}}^{2}$, preprint (2006) math.GT/0612130.

[3] A. Akhmedov \& B. Doug Park, Exotic Smooth Structures on Small 4-Manifolds, Invent. Math. 173 (2008), no. 1, 209-223, MR 2403396, Zbl 1144.57026.

[4] S. Baldridge, New symplectic 4-manifolds with $b_{+}=1$, Math. Ann. 333 (2005), 633-643, MR 2198801, Zbl 1082.53079.

[5] S. Baldridge \& P. Kirk, On symplectic 4-manifolds with prescribed fundamental group, Comment. Math. Helv. 82 (2007), no. 4, 845-875, MR 2341842, Zbl pre05229484.

[6] S. Baldridge \& P. Kirk, A symplectic manifold homemorphic but not diffeomorphic to $\mathbb{C P}^{2} \# 3 \overline{\mathbb{C P}}^{2}$, Geometry \& Topology 12 (2008), 919-940, MR 2403801, Zbl pre05275135.

[7] S. Baldridge \& P. Kirk, Symplectic 4-manifolds with arbitrary fundamental group near the Bogomolov-Miyaoka-Yau line, J. Symplectic Geom. 4 (2006), no. 1, 63-70, MR 2240212, Zbl 1105.53067.

[8] W. Barth, C. Peters \& A. Van de Ven, Compact complex surfaces, Ergebnisse der Mathematik und ihrer Grenzgebiete (3), 4. Springer-Verlag, Berlin, 1984. x+304 pp., MR 0749574, Zbl 0718.14023.

[9] J. F. Davis \& P. Kirk, Lecture notes in algebraic topology, Graduate Studies in Mathematics, 35, American Mathematical Society, Providence, RI, 2001. xvi+367 pp., MR 1841974, Zbl 1018.55001.

[10] R. Fintushel \& R. Stern, Surgery on nullhomolgous tori and simply connected 4-manifolds with $b^{+}=1$, J. Topol. 1 (2008), no. 1, 1-15, MR 2365649, Zbl pre05254778.

[11] R. Fintushel \& R. Stern, Knots, links, and 4-manifolds, Invent. Math. 134 (1998), no. 2, 363-400, MR 1650308, Zbl 0914.57015.

[12] R. Fintushel \& R. Stern, Families of simply connected 4-manifolds with the same Seiberg-Witten invariants, Topology 43 (2004), no. 6, 1449-1467, MR 2081432, Zbl 1064.57036.

[13] R. Fintushel \& R. Stern, Will we ever classify simply-connected smooth 4manifolds?, Clay Mathematics Proceedings 5 (2006), Floer Homology, Gauge Theory and Low Dimensional Topology, CMI/AMS Book Series, 225-240, MR 2233609, Zbl 1097.57001.

[14] M. Freedman, The topology of four-dimensional manifolds, J. Differential Geom. 17 (1982), no. 3, 357-453, MR 0679066, Zbl 0528.57011.

[15] R. Gompf, A new construction of symplectic manifolds, Ann. of Math. (2) 142 (1995), no. 3, 527-595, MR 1356781, Zbl 0849.53027. 
[16] R. Gompf \& A. Stipsicz, 4-manifolds and Kirby calculus, Graduate Studies in Mathematics, 20, American Mathematical Society, Providence, RI, 1999, xvi+558 pp., MR 1707327, Zbl 0933.57020.

[17] P. Kirk \& C. Livingston, The Hausmann-Weinberger 4-manifold invariant of abelian groups, Proc. Amer. Math. Soc. 133 (2005), no. 5, 1537-1546, MR 2111955, Zbl 1061.57001.

[18] P. Kirk \& C. Livingston, The geography problem for 4-manifolds with specified fundamental group, Trans. Amer. Math. Soc. 361 (2009), 4091-4124.

[19] D. Kotschick, On manifolds homeomorphic to $\mathbb{C P}^{2} \# 8 \overline{\mathbb{C P}}^{2}$, Invent. Math. 95 (1989), no. 3, 591-600, MR 0979367, Zbl 0691.57008.

[20] D. Kotschick, Minimizing Euler characteristics of symplectic four-manifolds, Proc. Amer. Math. Soc. 134 (2006), no. 10, 3081-3083, MR 2231635, Zbl 1094.57004.

[21] K. M. Luttinger, Lagrangian Tori in $\mathbb{R}^{4}$, J. Differential Geom. 42 (1995), no. 2, 220-228, MR 1366546, Zbl 0861.53029.

[22] D. McDuff \& D. Salamon, Introduction to symplectic topology, Second edition. Oxford Mathematical Monographs. The Clarendon Press, Oxford University Press, New York, 1998. x+486 pp., MR 1698616, Zbl 0844.58029.

[23] J. Morgan, T. Mrowka \& Z. Szabo, Product formulas along $T^{3}$ for SeibergWitten invariants, Math. Res. Lett. 4 (1997), no. 6, 915-929, MR 1492130, Zbl 0892.57021.

[24] B. Ozbagci \& A. Stipsicz, Noncomplex smooth 4-manifolds with genus 2 Lefschetz fibrations, Proc. Amer. Math. Soc. 128 (2000), no. 10, 3125-3128, MR 1670411, Zbl 0951.57015.

[25] P. Ozsváth \& Z. Szabó, On Park's exotic smooth four-manifolds, 'Geometry and topology of manifolds', 253-260, Fields Inst. Commun., 47, Amer. Math. Soc., Providence, RI, 2005, MR 2189937, Zbl 1095.57027.

[26] B. Doug Park, Exotic smooth structures on $3 \mathbb{C P}^{2} \# n \overline{\mathbb{C P}}^{2}$, Proc. Amer. Math. Soc. 128 (2000), no. 10, 3057-3065, MR 1664426, Zbl 0957.5702.

[27] Jongil Park, Simply connected symplectic 4-manifolds with $b_{2}^{+}=1$ and $c_{1}^{2}=2$, Invent. Math. 159 (2005), no. 3, 657-667, MR 2125736, Zbl 1080.57030.

[28] Jongil Park, The geography of symplectic 4-manifolds with arbitrary fundamental group, Proc. Amer. Math. Soc. 135 (2007), no. 7, 2301-2307, MR 2299508, Zbl 1116.57023.

[29] Jongil Park, A. Stipsicz \& Z. Szabó, Exotic smooth structures on $\mathbb{C P}^{2} \# 5 \overline{\mathbb{C P}^{2}}$, Math. Res. Lett. 12 (2005), no. 5-6, 701-712, MR 2189231, Zbl 1081.57025.

[30] T. Perutz, A remark on Kähler forms on symmetric products of Riemann surfaces, Preprint (2005) math.SG/0501547.

[31] D. Salamon, Removable singularities and a vanishing theorem for SeibergWitten invariants, Turkish J. Math 20 (1996), no. 1, 61-73, MR 1392663, Zbl 0865.57019.

[32] I. Smith, Symplectic geometry of Lefschetz fibrations, Dissertation, Oxford 1998.

[33] A. Stipsicz \& Z. Szabó, An exotic smooth structure on $\mathbb{C P}^{2} \# 6 \overline{\mathbb{C P}}^{2}$, Geom. Topol. 9 (2005), 813-832, MR 2140993.

[34] A. Stipsicz \& Z. Szabó, Small Exotic 4-manifolds with $b_{2}^{+}=3$, Bull. London Math. Soc. 38 (2006), 501-506, MR 2239045, Zbl 1094.57027. 
[35] Z. Szabó, Simply-connected irreducible 4-manifolds with no symplectic structures, Invent. Math. 132 (1998), no. 3, 457-466, MR 1625732, Zbl 0906.57014.

[36] C. Taubes, Seiberg-Witten and Gromov invariants, Geometry and physics (Aarhus, 1995), 591-601, Lecture Notes in Pure and Appl. Math., 184, Dekker, New York, 1997, MR 1423194, Zbl 0873.57017.

[37] C. Taubes, The Seiberg-Witten invariants and symplectic forms, Math. Res. Lett. 1 (1994), no. 6, 809-822, MR 1306023, Zbl 0853.57019.

[38] C. Taubes, SW $\Rightarrow$ Gr: from the Seiberg-Witten equations to pseudo-holomorphic curves, J. Amer. Math. Soc. 9 (1996), no. 3, 845-918, MR 1362874, Zbl 0867.53025.

[39] M. Usher, Minimality and symplectic sums, Int. Math. Res. Not. 2006, Art. ID 49857, 17 pp., MR 2250015, Zbl 1110.57017.

Department of Mathematics Louisiana State University

BAton Rouge, LA 70817

E-mail address: sbaldrid@math.lsu.edu

Mathematics Department

INDIANA UNIVERSITY

BLOOMINGTON, IN 47405

E-mail address: pkirk@indiana.edu 